\title{
Effelsberg 100-m polarimetric observations of a sample of compact steep-spectrum sources ${ }^{\star}$
}

\author{
F. Mantovani ${ }^{1}$, K.-H. Mack ${ }^{1}$, F. M. Montenegro-Montes ${ }^{1,2,3}$, A. Rossetti ${ }^{1}$, and A. Kraus ${ }^{4}$ \\ 1 Istituto di Radioastronomia - INAF, via Gobetti 101, 40129 Bologna, Italy \\ e-mail: fmantovani@ira.inaf.it \\ 2 Dpto. de Astrofísica. Universidad de La Laguna, Avda. Astrofísico Fco. Sánchez s/n, 38200 La Laguna (Tenerife), Spain \\ Instituto de Astrofísica de Canarias. C/ via Láctea s/n, 38200 La Laguna (Tenerife), Spain \\ 4 Max-Planck-Institut für Radioastronomie, Auf dem Hügel 69, 53121 Bonn, Germany
}

Received 9 February 2009 / Accepted 4 May 2009

\begin{abstract}
Aims. We completed observations with the Effelsberg 100-m radio telescope to measure the polarised emission from a complete sample of compact steep-spectrum sources and improve our understanding of the physical conditions inside and around regions of radio emission embedded in dense interstellar environments.

Methods. We observed the sources at four different frequencies, namely $2.64 \mathrm{GHz}, 4.85 \mathrm{GHz}, 8.35 \mathrm{GHz}$, and $10.45 \mathrm{GHz}$, making use of the polarimeters available at the Effelsberg telescope. We complemented these measurements with polarisation parameters at $1.4 \mathrm{GHz}$ derived from the NRAO VLA Sky Survey. Previous single dish measurements were taken from the catalogue of Tabara and Inoue.

Results. The depolarisation index DP was computed for four pairs of frequencies. A drop in the fractional polarisation appeared in the radio emission when observing at frequencies below $\sim 2 \mathrm{GHz}$. Rotation measures were derived for about $25 \%$ of the sources in the sample. The values, in the source rest frame, range from about $-20 \mathrm{rad} \mathrm{m}^{-2}$ found for 3C 138 to $3900 \mathrm{rad} \mathrm{m}^{-2}$ in $3 \mathrm{C} 119$. In all cases, the $\lambda^{2}$ law is closely followed.

Conclusions. The presence of a foreground screen as predicted by the Tribble model or with "partial coverage" as defined by ourselves can explain the polarimetric behaviour of the CSS sources detected in polarisation by the present observations. Indication of repolarisation at lower frequencies was found for some sources. A case of possible variability in the fractional polarisation is also suggested. The most unexpected result was found for the distribution of the fractional polarisations versus the linear sizes of the sources. Our results appear to disagree with the findings of Cotton and collaborators and Fanti and collaborators for the B3-VLA sample of CSS sources, the so-called "Cotton effect", i.e., a strong drop in polarised intensity for the most compact sources below a given frequency. This apparent contradiction may, however, be caused by the large contamination of the sample by quasars with respect to the B3-VLA.
\end{abstract}

Key words. radio continuum: general - radio continuum: ISM - polarization

\section{Introduction}

Measurements of the polarised emission from gigahertz-peaked spectrum (GPS) and Compact Steep-Spectrum (CSS) sources can provide important information about the physical conditions inside and around the region of radio emission. GPS/CSS sources are physically small objects with radio sizes smaller than $1 \mathrm{kpc}$ (essentially on the narrow line region size scale) and $15 \mathrm{kpc}$, respectively, that reside inside their host galaxies. The most widely accepted interpretation is that GPS/CSS galaxies are young radio sources (of ages $<10^{6} \mathrm{yr}$, Fanti et al. 1990). This view is supported by measurements of hot spot advance speeds (e.g., Polatidis \& Conway 2003) and by means of spectral ageing studies (Murgia et al. 1999). GPS sources, which are the youngest, will grow into CSS and eventually into classical extended radio sources. Consequently, the measurement of the physical properties of GPS and CSS sources can provide insight into the conditions at the birth of a powerful

\footnotetext{
* Tables 2-4 and Appendices are only available in electronic form at http://www . aanda. org
}

radio source and those of sources developing in dense interstellar environments (see Fanti et al. 1995; Readhead et al. 1996). The observations suggest that intrinsic distorsions in CSS sources are be caused by their interactions with dense and inhomogeneous gaseous environments. Asymmetries in terms of flux density, arm ratio, spectral index, and polarisation suggest that they are expanding through the dense inhomogeneous interstellar medium of their host galaxies (e.g., Saikia et al. 2003; Rossetti et al. 2006).

An effect on the synchrotron polarised emission produced by this magnetized thermal plasma is Faraday rotation, which is proportional to the product of electron density and the magnetic field component parallel to the direction of propagation integrated along the line of sight. The rotation measure (RM) is the amount of Faraday rotation expressed in $\mathrm{rad} \mathrm{m}^{-2}$. The Faraday rotation can unambiguously be determined from observations for at least three different wavelengths. Strong variations in Faraday rotation across the telescope beam will reduce or depolarise the observed fractional polarisation. This effect can often completely depolarise regions of emission. 
In many sources, the polarisation increases with increasing frequency when observed at similar angular resolutions. The fractional polarisations of CSS sources at $15 \mathrm{GHz}(6-7 \%)$ indeed tend to be higher than at $5 \mathrm{GHz}$ (1-3\%; van Breugel et al. 1984; Saikia et al. 1987). This suggests that large Faraday depths are responsible for the depolarisation between $15 \mathrm{GHz}$ and $5 \mathrm{GHz}$ rather than magnetic field geometry.

The magnetized plasma responsible for Faraday rotation and depolarisation can be situated either within the source, in a foreground screen, or in both. However, there are several indications, such as the magnitudes of the RMs, the total rotation of the electric vector position angle $>90^{\circ}$ without very high depolarisation, and the lack of correlation between high RMs in extragalactic sources and Galactic latitude (see for example the observations of Cygnus A by Dreher et al. 1987), that most of the observable effects are not internal to the source, but are produced in foreground material in the vicinity of the radio synchrotron source (e.g., Leahy 1990).

A model to describe the depolarisation behaviour in external screens has been discussed by Burn (1966) and later generalized by Tribble (1991) who considered the importance of the sizes of the individual Faraday cells in relation to the observing beam sizes. A variation in the ratio of these sizes can cause a more or less strong decline in the intrinsic polarisation percentage towards longer wavelengths. A successful application of the Tribble model, which implies the existence of a foreground screen, can be found in Fanti et al. (2004), who analysed multifrequency observations of the B3-VLA CSS sources (Fanti et al. 2001).

Many GPS/CSS sources have been observed mainly with radio interferometers. These investigations have provided very interesting results about the polarised state of the radio emission in GPS/CSS sources. A summary can be found in O'Dea (1998) and Cotton et al. (2003). However, apart from the B3-VLA sample (Fanti et al. 2001), most of the samples observed in polarisation so far (Lüdke et al. 1998; Akujor et al. 1995; van Breugel et al. 1984; Rossetti et al. 2008) contained subsets of sources whose incompleteness hamper statistical studies or which were observed at a single or dual frequency.

The set up of this paper is as follows. Sample selection, observations and data reduction are described in Sect. 2. Results from the observations are presented in Sect. 3 and discussed in Sect 4. In Sect. 5 we draw our conclusions. Plots of the fractional polarisation $m$ and rotation measures RM versus wavelength squared are compiled in Appendix 1. Comments about individual sources can be found in Appendix 2.

\section{Observations and data reduction}

\subsection{The sample}

The sample of CSS sources subject to the present investigation was constructed by Fanti et al. (1990) from the 3CR catalogue (Jenkins et al. 1977) and the Peacock \& Wall (1982, hereafter PW) sample. Since there is enough information about the radio structures and the spectral behaviour of the sources in the 3CR and PW catalogues, it is safe to assume that the sample contains all the CSS sources belonging to those catalogues with projected linear sizes less than $15 \mathrm{kpc}$, flux density at $178 \mathrm{MHz} \geq 10 \mathrm{Jy}$, $\log P_{178}>26.5 \mathrm{~W} \mathrm{~Hz}^{-1}$, and with $|b|>10^{\circ}, \delta>10^{\circ}$, and can thus be considered statistically complete.

We observed all the sources belonging to this sample with the Effelsberg 100-m radio telescope at four frequencies between $2.64 \mathrm{GHz}$ and $10.45 \mathrm{GHz}$ in a relatively short period of time
Table 1. Observing parameters and estimated integration times.

\begin{tabular}{lrrrr}
\hline \hline Centre frequency [GHz] & 2.64 & 4.85 & 8.35 & 10.45 \\
\hline Bandwidth [MHz] & 80 & 500 & 1100 & 300 \\
System Temp.(zenith) [K] & 17 & 27 & 22 & 53 \\
Scan length ['] & 16 & 12 & 8 & 6 \\
Scan speed ['/min] & 45 & 45 & 40 & 30 \\
\hline
\end{tabular}

(i.e., contemporaneously at the three higher frequencies, and 16 months later at $2.64 \mathrm{GHz}$ ). We choose this approach to avoid time variability effects when measuring the percentage of polarised flux density for each of these sources, the depolarisation indices, and the RMs with the aim of understanding more clearly the physical conditions in which CSS sources eventually expand to become larger sources.

Polarisation parameters at $1.4 \mathrm{GHz}$ were also derived for the full sample from the National Radio Astronomy Observatory Very Large Array Sky Survey (NVSS; Condon et al. 1998). Because of their small angular sizes, CSS sources are pointlike at the NVSS resolution. In the analysis of sources that were found polarised at two or more of the five frequencies above, we complemented the polarisation measurements with those listed by Tabara \& Inoue (1980).

For consistency with previous work, we used the cosmology $H_{0}=100 \mathrm{~km} \mathrm{~s}^{-1} \mathrm{Mpc}^{-1}$ and $q_{0}=0.5$.

\subsection{Observations}

The Effelsberg 100-m telescope was used to observe the complete sample of GPS/CSS sources constructed by Fanti et al. (1990). To minimize the occurrence of possible $n \pi$ ambiguities in the determination of RMs, we observed the sources at four independent frequencies, namely $2.64 \mathrm{GHz}, 4.85 \mathrm{GHz}, 8.35 \mathrm{GHz}$, and $10.45 \mathrm{GHz}$, making use of the polarimeters available at the Effelsberg telescope. The observations were carried out in the period January 26 to February 1, 2005 at $4.85 \mathrm{GHz}, 8.35 \mathrm{GHz}$, and $10.45 \mathrm{GHz}$ and June 24 to 26, 2006 at $2.64 \mathrm{GHz}$. Since all the target sources are point-like to the Effelsberg telescope beams, we used cross-scanning to determine the total intensity and polarisation characteristics. All sources in the sample are very bright, thus standard cross-scans along the azimuth and elevation axes were used, with 4 to 8 subscans, according to the source flux densities. Table 1 summarises the observing parameters. For further details about the observation mode and a description of the receivers, we refer to Montenegro-Montes et al. (2008), Klein et al. (2003), and references therein. The calibration sources 3C 286 and 3C 295 were regularly observed to correct for time-dependent gain instabilities and to bring our measurements onto an absolute flux density scale (Baars et al. 1977). The quasar 3C 286 was also used as a polarisation calibrator to obtain the polarisation degree $m$ and the polarisation angle $\chi$ in agreement with values in the literature (Tabara et al. 1980). The unpolarised planetary nebula NGC 7027 was also observed to estimate the instrumental polarisation. We found an instrumental polarisation $\left(p_{\text {instr }}\right)$ of $0.5 \%$ at $2.64 \mathrm{GHz}, 0.5 \%$ at $4.85 \mathrm{GHz}$, $0.3 \%$ at $8.35 \mathrm{GHz}$, and $0.8 \%$ at $10.45 \mathrm{GHz}$.

The measurement of flux densities from the single-dish cross-scans was done by fitting Gaussians to the signal of the polarimeter output channels (Stokes $I, Q$ and $U$ ) and identifying the Gaussian amplitudes with the flux densities $S_{I}, S_{Q}$, and $S_{U}$. For all sources with significant $S_{Q}$ and $S_{U}$ contributions, the polarised flux density $S_{\mathrm{P}}$, the degree of linear polarisation $m$, and the polarisation angle $\chi$ were computed. 
We consider three main contributions to the flux density error as in Klein et al. (2003). These are

(i) the calibration error $\Delta S_{\mathrm{c}}$, which is estimated to be the dispersion in the different observations of the flux density calibrators, i.e., about $2 \%$ at all observing frequencies;

(ii) the error introduced by noise, $\Delta S_{i} \sim 2 \mathrm{mJy}(i=I, Q, U)$, which is estimated from the noise at the scan edges;

(iii) the confusion error $\Delta S_{\text {conf }}$ caused by background sources within the beam area, estimated to be $1.5 \mathrm{mJy}$ at $2.64 \mathrm{GHz}$, $0.45 \mathrm{mJy}$ at $4.85 \mathrm{GHz}, 0.17 \mathrm{mJy}$ at $8.35 \mathrm{GHz}$, and $0.08 \mathrm{mJy}$ at $10.45 \mathrm{GHz}$ (Klein et al. 2003; the value at $8.35 \mathrm{GHz}$ was extrapolated from these existing measurements. The confusion limits can be neglected in the calculation of the total error in Stokes $Q$ and $U$.

These contribute to the total error in the following way:

$\Delta S_{i}=\sqrt{\left(S_{i} * \Delta S_{\mathrm{c}}\right)^{2}+\Delta S_{i}^{2}+\Delta S_{\mathrm{conf}}^{2}}$.

Since we are dealing with relatively bright targets, instrumental polarisation is an issue in many cases. This has been included in the error of the fractional polarisation in the following way:

$\Delta S_{\mathrm{P}}=\sqrt{\frac{\left(S_{Q} * \Delta S_{Q}\right)^{2}+\left(S_{U} * \Delta S_{U}\right)^{2}}{S_{\mathrm{P}}^{2}}+\left(p_{\text {instr }} * S_{I}\right)^{2}}$.

For all other errors we follow the definitions given by Klein et al. (2003). The errors associated with the position angles $\chi$ also account for the distribution, assumed to be Gaussian, in the values of $\chi$ obtained for the calibrator 3C 286 in the calibration process.

\subsection{Archival data}

The Effelsberg measurements were complemented with data of the NRAO VLA Sky Survey (NVSS) at $1.4 \mathrm{GHz}$ (Condon et al. 1998). In all these measurements, our targets are point-like to the corresponding beams, thus beam depolarising effects are avoided. The polarised flux densities in Table 2 are given for sources with polarised flux densities greater than three times the rms error. The rms uncertainty is computed following Eq. (49) in Condon et al. (1998)

$\sigma_{P}^{2} \approx 2 \sigma_{Q, U}^{2}+\epsilon_{\mathrm{P}}^{2} A_{\mathrm{P}}^{2}$

where $\epsilon_{\mathrm{p}}^{2}$ is the residual instrumental polarisation, which is about $0.12 \%$ for a large sample of sources stronger than $1 \mathrm{Jy}$ and $A_{\mathrm{P}}$ is the fitted peaked amplitude. In Table 3, we report the fractional polarisation values for these sources.

\section{Results from the observations}

In Table 2, we present both total and polarised flux densities derived by analysing the results of the observations completed with the Effelsberg 100-m radio telescope at the four frequencies plus those extracted from the NVSS. These measurements plus those derived from the literature are shown in Appendix 1.

\subsection{Percentage of polarised emission}

The sample constructed by Fanti et al. (1990) contains 47 sources. We considered them to be polarised when the intensity of the polarised emission is 3 times the rms error estimated source by source for the polarised emission. This provides in general lower limits of $3 \%$ at $2.64 \mathrm{GHz}, 2 \%$ at $4.85 \mathrm{GHz}, 1 \%$ at $8.35 \mathrm{GHz}$, and $1 \%$ at $10.45 \mathrm{GHz}$. Table 3 summarizes the values of the polarisation parameters for sources with polarised flux densities above the detection limits.

A high fraction of sources have polarised emission, if any, below the detection limits of our Effelsberg observations. We found that 22 of them are polarised at $10.45 \mathrm{GHz}, 16$ sources are polarised at $8.35 \mathrm{GHz}, 10$ sources are polarised at $4.85 \mathrm{GHz}$, and 10 sources are polarised at $2.64 \mathrm{GHz}$. The number of sources with detected polarised emission clearly decreases from high to low frequency. The percentage of polarised emission $m$ ranges between $\sim 6 \%$ and $\sim 12 \%$ for the most polarised sources $3 \mathrm{C} 138$, $3 \mathrm{C} 286$, and $3 \mathrm{C} 454$.

Because most of the sources in the sample have polarised emission below the detection limits of our observations, the median values of $m$ for the entire sample are below $3 \%$ at $2.64 \mathrm{GHz}$, below $2 \%$ at $4.85 \mathrm{GHz}$, below $1 \%$ at both $8.35 \mathrm{GHz}$ and $10.45 \mathrm{GHz}$, and below $1.58 \%$ at $1.4 \mathrm{GHz}$.

\subsection{Depolarisation index and rotation measure}

Table 4 summarizes the values of the depolarisation index and of the observed RM $\left(\mathrm{RM}_{\mathrm{obs}}\right)$ and source rest frame $\mathrm{RM}\left(\mathrm{RM}_{\mathrm{rf}}=\right.$ $\left.\mathrm{RM}_{\mathrm{obs}} \times(1+z)^{2}\right)$ both given in $\mathrm{rad} \mathrm{m}^{-2}$. The depolarisation index $\mathrm{DP}=m_{\mathrm{l}} / m_{\mathrm{h}}$, is defined to be the ratio of the percentages of polarised emission at the lower $\left(m_{1}\right)$ to the higher $\left(m_{\mathrm{h}}\right)$ frequency. The present observations complemented with those taken from Tabara \& Inoue (1980), allow us to determine the values of the RMs for 16 sources in the list. The observing frequencies of the Effelsberg plus NVSS observations are suitably separated for a proper determination of the RMs. In particular, they allow us to apply unambiguous $n \pi$ de-rotation to the observed polarisation E-vector position angle $\chi$ at the various frequencies. When applied, these rotations always yield a linear regression with a least squares fit very close to 1 , expect for an optimal fit, for the $\lambda^{2}$ rotation. We also note that there is no significant difference between the values derived from our Effelsberg observations and those from Tabara \& Inoue (1980), which were acquired about 30 years earlier.

\section{Discussion}

In the following we discuss trends of the depolarisation index, the achieved values of the rotation measure, and the distribution of the percentage of polarised emission versus the linear sizes of the observed sources.

\subsection{General trends}

The depolarisation index DP has been computed for four pairs of frequencies. The mean and the median values of the DP are reported in Table 5. Clearly a drop in the fractional polarisation appears in the radio emission at frequencies below $\sim 2 \mathrm{GHz}$. The number of polarised sources in our list of CSS sources drops from $46 \%$ at $10.45 \mathrm{GHz}$ to $23 \%$ at $2.64 \mathrm{GHz}$, and they have a median fractional polarisation of below $2 \%$ at $5.0 \mathrm{GHz}$ that agrees with previous findings (for example Saikia et al. 1987).

In Figs. A.1 to A.16 (Appendix 1), we plot the fractional polarisation values $m$ derived from our Effelsberg observations complemented with those extracted from the NVSS at $1.4 \mathrm{GHz}$ and those taken from the Tabara \& Inoue (1980) catalogue. The general trend is a quick decrease in the fractional polarisation with increasing wavelength. However, $m$ does not always drop to 
Table 5. The mean and the median values of the depolarisation index.

\begin{tabular}{lll}
\hline \hline Depolarisation index & $\mathrm{DP}_{\text {mean }}$ & $\mathrm{DP}_{\text {median }}$ \\
\hline $\mathrm{DP}_{8.35 / 10.45}$ & $0.98 \pm 0.15$ & $1.00_{-0.03}^{+0.02}$ \\
$\mathrm{DP}_{4.85 / 8.35}$ & $0.90 \pm 0.20$ & $0.91_{-0.006}^{+0.18}$ \\
$\mathrm{DP}_{2.64 / 4.85}$ & $1.11 \pm 0.33$ & $1.01_{-0.12}^{+0.09}$ \\
$\mathrm{DP}_{1.4 / 2.64}$ & $0.33 \pm 0.23$ & $0.32_{-0.14}^{+0.30}$ \\
\hline
\end{tabular}

zero at longer wavelengths $(\lambda>49 \mathrm{~cm})$ as predicted by the Burn (1966) model. The Burn model (dashed line) and the Tribble (1991) model (solid line) have been plotted in Figs. A.1 to A.16, according to Eqs. (1) and (2) in Fanti et al. (2004). For both models, we have adopted as the intrinsic fractional polarisation $m_{0}$ the one measured by us at $10.45 \mathrm{GHz}$. For the Tribble model, which assumes RM randomly distributed and a distribution of cell sizes, the ratio of the characteristic scale representing the largest cell scale to the observing beam is taken to be equal to 1 . With these assumptions, the model corresponds to the highest possible polarisation at long wavelengths, thus an upper limit to the expected fractional polarisation behaviour.

Very low fractional polarisation for sources belonging to this class is also found with interferometric observations. For example, Peck \& Taylor (2000) did not detect any linear polarisation with VLBI observations at $8.35 \mathrm{GHz}$ on a sample of 21 compact symmetric objects (CSOs are CSS sources that exhibit core and lobe emission on each side of the core). While this strengthens the assumption that beam depolarisation plays a minor rôle it also means that the polarisation characteristics of CSS sources are not yet fully clarified.

\subsection{Model fitting}

Rotation Measures were calculated for about 1/3 of the sources in our list making use of our own measurements, NVSS and Tabara \& Inoue data. The observed RMs were calculated for 16 sources with at least two measurements of the position angles achieved by Effelsberg and NVSS observations. Plots of the position angle of the electric vector in degrees versus the $\lambda^{2}$ in $\mathrm{m}^{2}$ are shown in Figs. A.1-A.16.

In all cases, the $\lambda^{2}$ law is closely followed for least squares estimates of the linear regression close to 1 . To search for possible variability, we determined the RM both from our new measurements only and from all available measurements, including those of Tabara \& Inoue, which were measured about 30 years ago. No significant difference was found.

The observing frequencies were suitably separated to enable an unambiguous rotation of the polarisation $\boldsymbol{E}$-vectors. By analysing the combination of RM and fractional polarisation, we measured $\lambda^{2}$ rotation and depolarisation for all sources, which corresponded to depolarisation produced inside the source, and rotation produced outside. However, since $\delta \chi>\pi$, we actually observed not mixed-in gas but a foreground screen. A revised Tribble model such as that proposed by Rossetti et al. (2008) with a "partial coverage" of the source of radio emission by NLRs may account for the depolarisation behaviour.

Comments are necessary in a few cases.

- 3C 67: the RM values calculated making use of the three position angles of Table 3 give an excellent least squares fit by means of linear regression after rotating by both +and $\pi$ the nominal value of $59^{\circ} .3$ obtained at $1.4 \mathrm{GHz}$, namely $69 \mathrm{rad} \mathrm{m}^{-2}$ and $-71 \mathrm{rad} \mathrm{m}^{-2}$. The last value is close to that

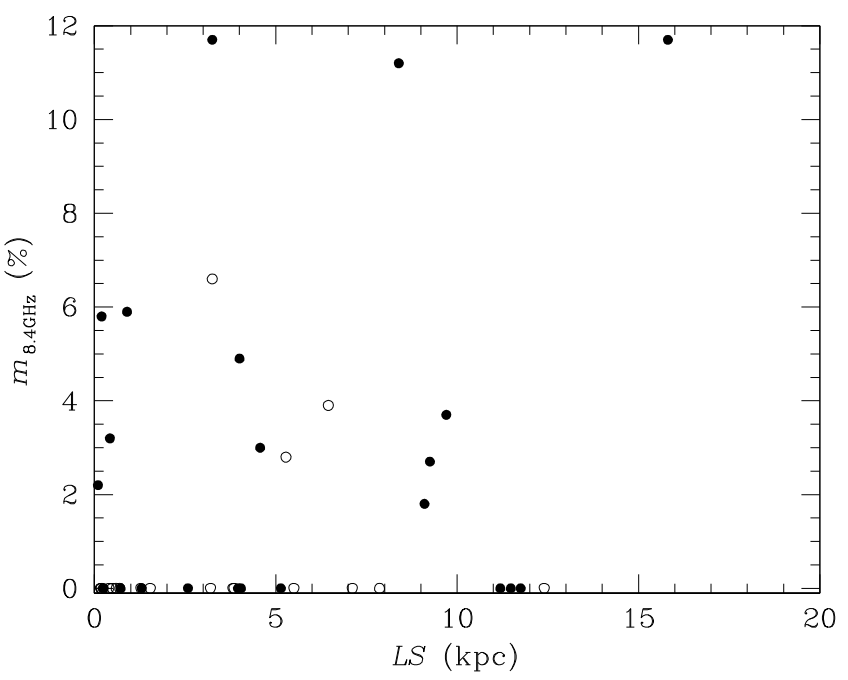

Fig. 1. Percentage of polarised emission versus source linear sizes at 8.35 GHz. Dots are Quasars; open circles are Galaxies.

$\left(-67 \mathrm{rad} \mathrm{m}^{-2}\right.$ ) calculated also using the RMs listed in the Tabara \& Inoue catalogue.

- 3C 138: the RM value calculated with the position angles listed in Table 3 is $-1 \mathrm{rad} \mathrm{m}^{-2}$ with a very poor mean least squares fit.

- 3C 286: the RM value is not given as the position angle of the electric vector $\left(33^{\circ}\right)$ for this source is taken as a reference to calibrate the position angles at all the observing frequencies.

- 3C 455: the least squares fit obtained with the values of Table 3 is worse than that achieved using all the available data.

In conclusion, we adopt in all cases the RMs listed in Col. 11 of Table 4 calculated using the present measurements of the position angles plus those taken from the catalogue by Tabara \& Inoue. The RMs in the source rest frames range between $-20 \mathrm{rad} \mathrm{m}^{-2}$ for $3 \mathrm{C} 138$ and $\sim 3900 \mathrm{rad} \mathrm{m}^{-2}$ for $3 \mathrm{C} 119$. Seven sources have a $\mathrm{RM}>400 \mathrm{rad} \mathrm{m}^{-2}$.

Examples of CSS sources with high values of $|\mathrm{RM}|\left(\geq 1000 \mathrm{rad} \mathrm{m}^{-2}\right.$ in the source rest frame) have been found in the past (O'Dea 1998, and references therein).

Three of them are in our list with measured integrated RMs, namely 3C 119, 1442+101 and 3C 318 for which we measured $3900 \mathrm{rad} \mathrm{m}^{-2},-1450 \mathrm{rad} \mathrm{m}^{-2}$, and $2260 \mathrm{rad} \mathrm{m}^{-2}$ respectively. For the source 1442+101 (OQ172), observed by Udomprasert (1998) with the VLBA, RMs up to $22400 \mathrm{rad} \mathrm{m}^{-2}$ were found. For 3C 119, Kato et al. (1987) found $3400 \mathrm{rad} \mathrm{m}^{-2}$ using single dish measurements. For 3C 318, Taylor et al. (1992) determined a RM of $1400 \mathrm{rad} \mathrm{m}^{-2}$ using VLA observations. There are however CSS sources in our sample with smaller values of RM.

As described in more detail in Appendix 2, the source 3C 298 shows evidence of temporal variability in the fractional polarisation.

\subsection{The "Cotton effect"}

As an example, we show the percentage of polarised emission versus the source's linear size in kpc at a frequency of $8.35 \mathrm{GHz}$ in Fig. 1. Linear dimensions for sources labelled with "**" in Table 4, for which $z$ became available after 1990, were calculated. We note that in the plot sources with linear sizes greater than $15 \mathrm{kpc}$ are omitted and sources with polarised emission below the detection limits are plotted assuming $m=0$. 
The most unexpected result that we find is the distribution of points drawn in the plots at the Effelsberg and the NVSS observing frequencies. Polarised sources are distributed all over the $m-L S$ space. This seems to clash with the findings of Cotton et al. (2003) and Fanti et al. (2004) for the B3-VLA sample of CSS sources. Cotton et al. (2003) making use of NVSS at $1.4 \mathrm{GHz}$ found that sources smaller than $6 \mathrm{kpc}$ are weakly polarised, and that polarised sources have linear sizes greater than $6 \mathrm{kpc}$ ("Cotton effect"). Both the jet and the counter-jet are included in the source linear size. This implies a drastic change in the interstellar medium at about $3 \mathrm{kpc}$. This result was later confirmed by Fanti et al. (2004), who observed the same sample with the VLA at $4.9 \mathrm{GHz}$ and $8.5 \mathrm{GHz}$ and with the WSRT at $2.64 \mathrm{GHz}$ (Rossetti et al. 2008).

The main difference between the two samples, i.e., the B3-VLA and the present 3CR\&PW sample of CSS sources, is represented by the number of quasars, 5-10\% and $49 \%$, respectively. Excluding the quasars, we find that the Cotton effect is clearly evident again. However, we also note three outliers, the galaxies 3C 93.1, 3C 268.3, and 3C 318. On the other hand, it has been found by Rossetti et al. (2008) that the Cotton effect is not obeyed by all sources. This indicates that the current depolarisation scenarios might not fully explain the observed behaviour. This is also further indication that CSS sources optically identified with quasars may represent a separate class of objects.

\section{Conclusions}

With the present observations of a complete sample of CSS sources at 4 different frequencies made with the Effelsberg 100-m radio telescope and from archived NVSS data, the following results have been reached:

a) It is confirmed that CSS sources are weakly polarised, low values of the median fractional polarisation being found. Where $m$ can be compared with those obtained by Klein et al. (2003) for the steep spectrum extended radio sources selected in the B3-VLA sample, larger values are found, which are $2.2 \%$ at $1.4 \mathrm{GHz}, 3.7 \%$ at $2.64 \mathrm{GHz}, 5.2 \%$ at $4.85 \mathrm{GHz}$, and $5.8 \%$ at $10.45 \mathrm{GHz}$. The more sensitive VLA observations at $1.4 \mathrm{GHz}$ of the CSS sources in the $3 \mathrm{CR} \& \mathrm{PW}$ sample extracted from the NVSS also show a median value for the fractional polarisation of $\sim 1.6 \%$, which is rather low.

b) As expected, the percentage of polarised sources decreases from higher to lower frequencies. We found 22 sources polarised at $10.45 \mathrm{GHz}, 16$ sources polarised at $8.35 \mathrm{GHz}$, 10 sources polarised at both $4.85 \mathrm{GHz}$ and $2.64 \mathrm{GHz}$, and 13 at $1.4 \mathrm{GHz}$. In general, the percentage of polarised emission remains almost constant down to $2.64 \mathrm{GHz}$, then drastically drops at frequencies below $\sim 2 \mathrm{GHz}$. There may be cases of repolarisation. Of particular interest, if this finding is confirmed, is the case of $3 \mathrm{C} 455$.

c) A case of variability with time of the fractional polarisation is suggested for the source 3C 298.

d) The data obtained with these observations allowed us to determine the RMs for 16 sources. The RMs in the source rest frames range between $-20 \mathrm{rad} \mathrm{m}^{-2}$ for 3C 138 and $\sim 3900 \mathrm{rad} \mathrm{m}^{-2}$ for 3C 119. Seven sources show a RM > $400 \mathrm{rad} \mathrm{m}^{-2}$, confirming the high values of the RM usually found for CSS sources.

e) In all cases the $\lambda^{2}$ law is followed closely. The observing frequencies were suitably separated to enable an unambiguous rotation of the polarisation $\boldsymbol{E}$-vectors. Analysing the combination of RM and fractional polarisation, we identified $\lambda^{2}$ rotation and depolarisation for all sources. A revised Tribble model such as that proposed by Rossetti et al. (2008) with a "partial coverage" of the source of radio emission by NLRs may account for the depolarisation behaviour.

f) The $m-L S$ diagram contains sources polarised at any linear size and at all available frequencies, including the NVSS 1.4 GHz data, i.e., also for sources smaller than $6 \mathrm{kpc}$ in linear size, in contrast to the so-called "Cotton effect" later confirmed by Fanti et al. (2004) for the B3-VLA sample of CSS sources. However, plotting $m$ versus $L S$ for objects optically identified with galaxies, the so-called "Cotton effect" is reproduced. Discussing the interesting, large difference in the percentage of objects identified as quasars in the two samples was not a subject of the present investigation.

g) CSS sources optically identified with quasars may represent a separate class of objects.

Acknowledgements. This work is based on observations with the 100-m telescope of the MPIfR (Max-Planck-Institut für Radioastronomie) at Effelsberg. It has benefited from research funding from the European Community's sixth Framework Programme under RadioNet R113CT 2003 5058187. F.M. likes to thank Prof. Anton Zensus, Director, for the kind hospitality at the MaxPlanck-Institut für Radioastronomie, Bonn, for a period during which part of this work was done. The National Radio Astronomy Observatory is a facility of the National Science Foundation operated under cooperative agreement by Associated Universities, Inc. We are very grateful to an anonymous referee for very helpful comments and suggestions and for a careful reading of the manuscript of this paper.

\section{References}

Akujor, C. E., \& Garrington, S. T. 1995, A\&AS, 112, 235

Aller, M. F., Aller, H. D., \& Huges, P. A. 2003, ApJ, 518, 33

Baars, J. W. M., Genzel, R., Pauliny-Toth, I. I. K., \& Witzel, A. 1977, A\&A, 61, 99

Burn, B. J. 1966, MNRAS, 133, 67

Cohen, A. S., Lane, W. M., Cotton, W. D., et al. 2007, AJ, 134, 1245

Condon, J. J., Cotton, W. D., Greisen, E. W., et al. 1998, AJ, 115, 1693

Cotton, W. D., Dallacasa, D., Fanti, C., et al. 2003, PASA, 20, 12

Dreher, J. W., Carilli, C. L., \& Perley, R. A. 1987, ApJ, 316, 611

Fanti, R., Fanti, C., Schilizzi, R. T., et al. 1990, A\&A, 231, 333

Fanti, C., Fanti, R., Dallacasa, D., et al. 1995, A\&A, 302, 317

Fanti, C., Pozzi, F., Dallacasa, D., et al. 2001, A\&A, 369, 380

Fanti, C., Branchesi, M., Cotton, W. D., et al. 2004, A\&A, 427, 465

Hes, R., Barthel, P. D., \& Fosbury, R. A. E. 1996, A\&A, 313, 423

Jenkins, C. J., Pooley, G. G., \& Riley, J. M. 1977, MNRAS, 84, 61

Kato, T., Tabara, H., Inoue, M., \& Aizu, K. 1987, Nature, 329, 223

Klein, U., Mack, K.-H., Gregorini, L., \& Vigotti, M. 2003, A\&A, 406, 579

Labiano, A., Barthel, P. D., O’Dea, C. P., et al. 2007, A\&A, 463, 97

Laing, R. 1984, Proceeding NRAO Workshop, 9, 90

Leahy, J. P. 1990, Parsec-Scale Radio Jets, ed. J. A. Zensus, \& T. J. Pearson (Cambridge: Cambridge University Press)

Montenegro-Montes, F. M., Mack, K.-H., Vigotti, M., et al. 2008, MNRAS, 388, 1853

Murgia, M., Fanti, C., Fanti, R., et al. 1999, A\&A, 345, 769

O'Dea, C. 1998, PASP, 110, 493

Peacock, J. A., \& Wall, J. V. 1982, MNRAS, 198, 843

Peck, A. B., \& Taylor, G. B. 2000, ApJ, 534, 90

Polatidis, A. CG., \& Conway, J. E. 2003, PASA, 20, 69

Readhead, A. C. S., Taylor, G. B., Xu, W., et al. 1996, ApJ, 460, 612

Rossetti, A., Mantovani, F., Dallacasa, D., et al. 2005, A\&A, 434, 449

Rossetti, A., Fanti, C., Fanti, R., et al. 2006, A\&A, 449, 49

Rossetti, A., Dallacasa, D., Fanti, C., et al. 2008, A\&A, 487, 865

Saikia, D. J., \& Gupta, N. 2003, A\&A, 405, 499

Saikia, D. J., Singal, A. K., \& Cornwell, T. J. 1987, MNRAS, 224, 379

Stanghellini, C., O’Dea, C. P., Baum, S. A., \& Laurikainen, E. 1993, ApJS, 88, 1

Stanghellini, C., O'Dea, C. P., Dallacasa, D., et al. 2005, A\&A, 443, 891

Tabara, H., \& Inoue, M. 1980, A\&AS, 39, 373

Taylor, G. B., Inoue, M., \& Tabara, X. 1992, A\&A, 264, 421

Tribble, P. C. 1991, MNRAS, 250, 726

Udomprasert, P. S., Taylor, G. B., Pearson, T. J., \& Roberts, D. H. 1997, ApJ, 483, L9

van Breugel, W., Miley, G., \& Heckman, T. 1984, ApJ, 276, 79

Willott, C. J., Rawlings, S., Blundell, K. M., \& Lacy, M. 1998, MNRAS, 300, 625 
F. Mantovani et al.: Effelsberg polarimetry of CSS sources, Online Material $p 1$

Table 2. Flux densities and polarised flux densities from Effelsberg 100-m and NVSS measurements.

\begin{tabular}{|c|c|c|c|c|c|c|c|c|c|c|c|}
\hline Name & $\begin{array}{c}S_{1.4} \\
{[\mathrm{mJy}]}\end{array}$ & $\begin{array}{c}S_{2.64} \\
{[\mathrm{mJy}]}\end{array}$ & $\begin{array}{c}S_{4.85} \\
{[\mathrm{mJy}]}\end{array}$ & $\begin{array}{c}S_{8.35} \\
{[\mathrm{mJy}]}\end{array}$ & $\begin{array}{l}S_{10.45} \\
{[\mathrm{mJy}]}\end{array}$ & $\begin{array}{c}S_{\mathrm{p} 1.4} \\
{[\mathrm{mJy}]}\end{array}$ & $\begin{array}{l}S_{\mathrm{p} 2.64} \\
{[\mathrm{mJy}]}\end{array}$ & $\begin{array}{l}S_{\mathrm{p} 4.85} \\
{[\mathrm{mJy}]}\end{array}$ & $\begin{array}{l}S_{\mathrm{p} 8.35} \\
{[\mathrm{mJy}]}\end{array}$ & $\begin{array}{c}S_{\mathrm{p} 10.45} \\
{[\mathrm{mJy}]}\end{array}$ & \\
\hline $3 \mathrm{C} 43$ & $0127+23$ & $29414 \pm 88$ & $1787 \pm 37$ & $1109 \pm 22$ & $698 \pm 14$ & $578 \pm 12$ & $<10.8$ & $<38.4$ & $<6.6$ & $14.7 \pm 4.1$ & $12.2 \pm 2.3$ \\
\hline $3 \mathrm{C} 48$ & $0134+32$ & $16018 \pm 481$ & $9373 \pm 191$ & $5534 \pm 113$ & $3243 \pm 65$ & $2495 \pm 52$ & $70.8 \pm 57.6$ & $<195.0$ & $185.5 \pm 43.1$ & $160.5 \pm 18.8$ & $139.5 \pm 5.6$ \\
\hline 3C 49 & $0138+13$ & $2740 \pm 82$ & $1552 \pm 33$ & $878 \pm 18$ & $494 \pm 10$ & $376 \pm 8$ & $<9.9$ & $<34.5$ & $<20.4$ & $<8.7$ & $<6.3$ \\
\hline $3 \mathrm{C} 67$ & $0221+27$ & $3024 \pm 91$ & $1757 \pm 36$ & $997 \pm 20$ & $575 \pm 12$ & $419 \pm 9$ & $17.0 \pm 10.9$ & $<37.8$ & $<23.4$ & $22.3 \pm 3.4$ & $15.7 \pm 1.9$ \\
\hline 4C 34.07 & $0223+34$ & $2894 \pm 87$ & $2483 \pm 51$ & $2234 \pm 46$ & $1452 \pm 30$ & $1212 \pm 25$ & $<10.5$ & $<51.6$ & $<52.2$ & $<24.9$ & $<13.2$ \\
\hline \multirow[t]{2}{*}{ 4C 16.09} & $0316+16$ & $8028 \pm 241$ & $5038 \pm 103$ & $2937 \pm 60$ & $1563 \pm 32$ & $1187 \pm 25$ & $<28.9$ & $<103.5$ & $<6.9$ & $<27.0$ & $<12.9$ \\
\hline & $0319+12$ & $1907 \pm 67$ & $1704 \pm 35$ & $1791 \pm 37$ & $1527 \pm 31$ & $1549 \pm 32$ & $71.8 \pm 7.0$ & $95.3 \pm 12.3$ & $113.0 \pm 14.1$ & $88.9 \pm 8.9$ & $77.2 \pm 5.7$ \\
\hline 3C 93.1 & $0345+33$ & $2365 \pm 71$ & $1390 \pm 29$ & $926 \pm 19$ & $477 \pm 10$ & $383 \pm 8$ & $<8.6$ & $<30.0$ & $<21.6$ & $<8.4$ & $9.1 \pm 1.6$ \\
\hline \multirow[t]{2}{*}{ 4C 76.03} & $0404+76$ & $5619 \pm 169$ & $4042 \pm 83$ & $3355 \pm 69$ & $2087 \pm 42$ & $1648 \pm 34$ & $<20.3$ & $<83.4$ & $<78.3$ & $<36.0$ & $<18.0$ \\
\hline & $0428+20$ & $3755 \pm 112$ & $3166 \pm 65$ & $2761 \pm 57$ & $1542 \pm 31$ & $1289 \pm 26$ & $<13.6$ & $<65.7$ & $<64.5$ & $<26.7$ & $<14.1$ \\
\hline 3C 119 & $0429+41$ & $9832 \pm 295$ & $6126 \pm 126$ & $4722 \pm 97$ & $2677 \pm 54$ & $2260 \pm 47$ & $<35.4$ & $<125.4$ & $<110.1$ & $157.9 \pm 15.7$ & $200.6 \pm 8.9$ \\
\hline 3C 138 & $0518+165$ & $8603 \pm 258$ & $5780 \pm 119$ & $4119 \pm 84$ & $2552 \pm 53$ & $2160 \pm 45$ & $619.0 \pm 31.0$ & $595.5 \pm 41.0$ & $411.6 \pm 32.8$ & $297.8 \pm 17.6$ & $218.8 \pm 8.8$ \\
\hline $3 \mathrm{C} 147$ & $0538+49$ & $22880 \pm 686$ & $13436 \pm 276$ & $10120 \pm 207$ & $4735 \pm 96$ & $3823 \pm 80$ & $<82.3$ & $<274.5$ & $<236.1$ & $<81.6$ & $47.3 \pm 13.6$ \\
\hline $3 \mathrm{C} 186$ & $0740+38$ & $1236 \pm 37$ & $615 \pm 14$ & $271 \pm 8$ & $114 \pm 3$ & $85 \pm 3$ & $<4.6$ & $<15.3$ & $<6.3$ & $<2.4$ & $<3.0$ \\
\hline $3 \mathrm{C} 190$ & $0758+14$ & $2734 \pm 82$ & $1359 \pm 28$ & $746 \pm 16$ & $426 \pm 10$ & $360 \pm 8$ & $<9.9$ & $<31.5$ & $<17.4$ & $<7.8$ & $<5.1$ \\
\hline $3 \mathrm{C} 216$ & $0906+43$ & $4239 \pm 127$ & $1446 \pm 50$ & $1731 \pm 35$ & $1413 \pm 29$ & $1337 \pm 29$ & $<15.3$ & $<52.5$ & $<40.5$ & $<24.3$ & $<14.7$ \\
\hline 3C 237 & $1005+07$ & $6522 \pm 196$ & $3608 \pm 74$ & $1971 \pm 42$ & $1034 \pm 21$ & $823 \pm 17$ & $<23.5$ & $<76.5$ & $<45.9$ & $<18.0$ & $9.7 \pm 3.2$ \\
\hline $3 \mathrm{C} 241$ & $1019+22$ & $1686 \pm 51$ & $799 \pm 17$ & $350 \pm 7$ & $161 \pm 33$ & $112 \pm 3$ & $<6.2$ & $<19.5$ & $<8.1$ & $<3.0$ & $<1.2$ \\
\hline 4C 31.38 & $1153+31$ & $2978 \pm 89$ & $1751 \pm 36$ & $1030 \pm 21$ & $545 \pm 11$ & $441 \pm 10$ & $<10.8$ & $<45.0$ & $<24.0$ & $<9.6$ & $8.6 \pm 1.8$ \\
\hline \multirow[t]{2}{*}{$3 \mathrm{C} 268.3$} & $1203+64$ & $3719 \pm 112$ & $1947 \pm 39$ & $1153 \pm 23$ & $618 \pm 13$ & $479 \pm 10$ & $<13.4$ & $<40.8$ & $<27.0$ & $17.2 \pm 3.7$ & $14.2 \pm 2.1$ \\
\hline & $1225+36$ & $2098 \pm 63$ & $1434 \pm 30$ & $753 \pm 16$ & $353 \pm 9$ & $239 \pm 5$ & $<7.7$ & $<31.5$ & $<17.7$ & $<6.3$ & $<2.4$ \\
\hline 3C 277.1 & $1250+56$ & $2288 \pm 69$ & $1343 \pm 30$ & $790 \pm 16$ & $481 \pm 10$ & $384 \pm 8$ & $<8.3$ & $<29.4$ & $<18.3$ & $14.3 \pm 2.9$ & $9.9 \pm 1.8$ \\
\hline 4C 32.44 & $1323+32$ & $4862 \pm 146$ & $3369 \pm 70$ & $2293 \pm 47$ & $1540 \pm 31$ & $1293 \pm 28$ & $<17.5$ & $17.3 \pm 2.3$ & $<53.4$ & $<26.7$ & $<14.1$ \\
\hline 3C 286 & $1328+30$ & $14902 \pm 447$ & $10607 \pm 219$ & $7430 \pm 152$ & $5179 \pm 104$ & $4474 \pm 94$ & $999.9 \pm 53.7$ & $1152.7 \pm 74.1$ & $776.7 \pm 59.4$ & $607.4 \pm 31.5$ & $525.8 \pm 18.4$ \\
\hline $3 \mathrm{C} 287$ & $1328+27$ & $7052 \pm 212$ & $4697 \pm 97$ & $3130 \pm 64$ & $2050 \pm 42$ & $1748 \pm 36$ & $45.6 \pm 25.4$ & $168.7 \pm 32.3$ & $116.6 \pm 24.4$ & $65.2 \pm 11.8$ & $58.6 \pm 6.4$ \\
\hline \multirow[t]{2}{*}{$4 \mathrm{C} 62.22$} & $1358+62$ & $4308 \pm 129$ & $2765 \pm 57$ & $1701 \pm 35$ & $1080 \pm 22$ & $888 \pm 18$ & $<15.6$ & $<57.3$ & $<39.6$ & $<18.6$ & $<9.9$ \\
\hline & $1413+34$ & $1864 \pm 56$ & $1488 \pm 30$ & $1059 \pm 22$ & $735 \pm 15$ & $621 \pm 14$ & $<6.8$ & $45.8 \pm 11.2$ & $<24.6$ & $<12.9$ & $<7.5$ \\
\hline 3C 298 & $1416+06$ & $6100 \pm 183$ & $2888 \pm 60$ & $1432 \pm 30$ & $789 \pm 17$ & $616 \pm 14$ & $<22.0$ & $<60.0$ & $<33.3$ & $14.6 \pm 4.6$ & $12.8 \pm 2.6$ \\
\hline 3C 299 & $1419+41$ & $3147 \pm 111$ & $1721 \pm 35$ & $922 \pm 20$ & $502 \pm 10$ & $400 \pm 9$ & $<11.4$ & $<40.8$ & $<21.6$ & $<9.0$ & $<6.6$ \\
\hline OQ172 & $1442+10$ & $2418 \pm 72$ & $1398 \pm 38$ & $1032 \pm 21$ & $641 \pm 14$ & $516 \pm 11$ & $31.7 \pm 8.8$ & $63.2 \pm 12.0$ & $26.5 \pm 8.0$ & $14.2 \pm 3.8$ & $<8.7$ \\
\hline $3 \mathrm{C} 303.1$ & $1443+77$ & $1880 \pm 66$ & $882 \pm 19$ & $413 \pm 8$ & $199 \pm 4$ & $128 \pm 3$ & $<6.9$ & $<20.1$ & $<9.6$ & $<3.9$ & $<2.4$ \\
\hline 3C 305.1 & $1447+77$ & $1666 \pm 50$ & $837 \pm 18$ & $412 \pm 9$ & $208 \pm 4$ & $154 \pm 3$ & $<6.2$ & $<20.1$ & $<9.6$ & $<11.7$ & $<3.9$ \\
\hline 3С 309.1 & $1458+71$ & $7468 \pm 224$ & $5129 \pm 105$ & $3552 \pm 73$ & $2568 \pm 52$ & $2196 \pm 46$ & $91.1 \pm 26.9$ & $188.3 \pm 35.2$ & $8.5 \pm 2.8$ & $70.8 \pm 14.8$ & $55.5 \pm 8.8$ \\
\hline \multirow[t]{2}{*}{$3 \mathrm{C} 318$} & $1517+20$ & $2688 \pm 81$ & $1417 \pm 29$ & $764 \pm 16$ & $417 \pm 8$ & $322 \pm 7$ & $<9.8$ & $<30.9$ & $25.8 \pm 6.0$ & $27.6 \pm 2.6$ & $29.6 \pm 1.8$ \\
\hline & $1600+33$ & $2991 \pm 90$ & $1980 \pm 41$ & $1433 \pm 29$ & $1049 \pm 21$ & $915 \pm 20$ & $<10.8$ & $<42.3$ & $<33.3$ & $<18.3$ & $<10.5$ \\
\hline OS111 & $1607+26$ & $4908 \pm 147$ & $3140 \pm 65$ & $1728 \pm 35$ & $902 \pm 18$ & $681 \pm 15$ & $<17.7$ & $<98.7$ & $<40.2$ & $<15.6$ & $<8.1$ \\
\hline $3 \mathrm{C} 343$ & $1634+62$ & $5002 \pm 150$ & $2858 \pm 59$ & $1503 \pm 31$ & $808 \pm 16$ & $622 \pm 13$ & $<18.0$ & $<59.1$ & $<35.1$ & $<14.1$ & $8.4 \pm 2.5$ \\
\hline $3 \mathrm{C} 343.1$ & $1637+62$ & $4611 \pm 138$ & $2384 \pm 49$ & $1190 \pm 24$ & $631 \pm 13$ & $479 \pm 10$ & $<16.6$ & $<49.2$ & $<27.9$ & $<11.1$ & $<6.0$ \\
\hline 3C 346 & $1641+17$ & $3666 \pm 110$ & $2260 \pm 47$ & $1426 \pm 29$ & $915 \pm 19$ & $773 \pm 17$ & $80.6 \pm 13.3$ & $<47.7$ & $<33.3$ & $<45.9$ & $13.8 \pm 3.0$ \\
\hline 4C 39.56 & $1819+39$ & $3507 \pm 105$ & $1871 \pm 42$ & $938 \pm 19$ & $463 \pm 10$ & $337 \pm 7$ & $<12.7$ & $<39.9$ & $<21.9$ & $<8.1$ & $<4.5$ \\
\hline $3 \mathrm{C} 380$ & $1828+48$ & $13573 \pm 413$ & $8139 \pm 167$ & $5073 \pm 105$ & $3523 \pm 71$ & $3055 \pm 63$ & $62.2 \pm 49.5$ & $<166.5$ & $<118.2$ & $<60.6$ & $<32.7$ \\
\hline 4C 29.56 & $1829+29$ & $2924 \pm 88$ & $1945 \pm 40$ & $1168 \pm 24$ & $679 \pm 14$ & $533 \pm 12$ & $<10.6$ & $<40.8$ & $<27.3$ & $<12.0$ & $<6.9$ \\
\hline 4C 11.69 & $2230+11$ & $7202 \pm 216$ & $5695 \pm 118$ & $4135 \pm 85$ & $3396 \pm 69$ & $3209 \pm 67$ & $114.0 \pm 26.0$ & $229.6 \pm 39.1$ & $147.6 \pm 32.3$ & $127.3 \pm 19.6$ & $116.4 \pm 11.6$ \\
\hline $3 \mathrm{C} 454$ & $2249+18$ & $2133 \pm 64$ & $1265 \pm 26$ & $761 \pm 16$ & $469 \pm 10$ & $383 \pm 8$ & $77.4 \pm 7.8$ & $73.6 \pm 9.1$ & $71.0 \pm 6.1$ & $52.7 \pm 2.9$ & $42.8 \pm 1.9$ \\
\hline $3 \mathrm{C} 454.1$ & $2248+71$ & $1555 \pm 47$ & $696 \pm 15$ & $287 \pm 6$ & $119 \pm 3$ & $87 \pm 2$ & $<5.8$ & $<16.8$ & $<6.9$ & $<3.6$ & $<0.9$ \\
\hline \multirow[t]{2}{*}{$3 \mathrm{C} 455$} & $2252+12$ & $2706 \pm 81$ & $1491 \pm 30$ & $772 \pm 16$ & $421 \pm 93$ & $312 \pm 7$ & $55.8 \pm 9.9$ & $50.5 \pm 10.9$ & $<18.0$ & $<7.8$ & $4.7 \pm 1.5$ \\
\hline & $2342+82$ & $3777 \pm 113$ & $2275 \pm 47$ & $1308 \pm 27$ & $747 \pm 15$ & $579 \pm 12$ & $<13.7$ & $<47.4$ & $<30.6$ & $<13.2$ & $<6.6$ \\
\hline
\end{tabular}

The values at the various observing frequencies (in GHz) are organised as follows: Col. 1, source name; Col. 2, other name; Cols. 3 to 7 , flux density $S$; Cols. 8 to 12 , polarised flux density $S_{\mathrm{p}}$. 
F. Mantovani et al.: Effelsberg polarimetry of CSS sources, Online Material p 2

Table 3. Percentage of polarised flux density and position angle of the electric vector at the five frequencies.

\begin{tabular}{|c|c|c|c|c|c|c|c|c|c|c|c|c|}
\hline Name & & $\begin{array}{c}L S \\
{[\mathrm{kpc}]}\end{array}$ & $\begin{array}{c}m_{1.4} \\
{[\%]}\end{array}$ & $\begin{array}{r}m_{2.64} \\
{[\%]}\end{array}$ & $\begin{array}{r}m_{4.85} \\
{[\%]}\end{array}$ & $\begin{array}{r}m_{8.35} \\
{[\%]}\end{array}$ & $\begin{array}{r}m_{10.45} \\
{[\%]}\end{array}$ & $\begin{array}{r}\chi_{1.4} \\
{[\mathrm{deg}]}\end{array}$ & $\begin{array}{l}\chi_{2.64} \\
{[\mathrm{deg}]}\end{array}$ & $\begin{array}{l}\chi_{4.85} \\
{[\mathrm{deg}]}\end{array}$ & $\begin{array}{l}\chi_{8.35} \\
{[\mathrm{deg}]}\end{array}$ & $\begin{array}{l}\chi_{10.45} \\
\text { [deg] }\end{array}$ \\
\hline $3 \mathrm{C} 43$ & $0127+23$ & 12.85 & & & & $2.1 \pm 0.6$ & $2.1 \pm 0.4$ & & & & $2 \pm 2$ & $13 \pm 3$ \\
\hline $3 \mathrm{C} 48$ & $0134+32$ & 4.00 & $0.44 \pm 0.12$ & & $3.4 \pm 0.8$ & $4.9 \pm 0.6$ & $5.6 \pm 0.4$ & $-70.8 \pm 0.1$ & & $-78 \pm 2$ & $-68 \pm 2$ & $-61 \pm 2$ \\
\hline $3 \mathrm{C} 49$ & $0138+13$ & 3.85 & & & & & & & & & & \\
\hline $3 \mathrm{C} 67$ & $0221+27$ & 6.45 & $0.56 \pm 0.12$ & & & $3.9 \pm 0.6$ & $3.7 \pm 0.5$ & $59.3 \pm 0.5$ & & & $62 \pm 2$ & $62 \pm 3$ \\
\hline 4C 34.07 & $0223+34$ & $4.04 *$ & & & & & & & & & & \\
\hline \multirow[t]{2}{*}{ 4C 16.09} & $0316+16$ & $1.3^{+}$ & & & & & & & & & & \\
\hline & $0319+12$ & $0.2^{+}$ & $3.80 \pm 0.18$ & $5.6 \pm 0.7$ & $6.3 \pm 0.8$ & $5.8 \pm 0.6$ & $5.0 \pm 0.4$ & $41.0 \pm 0.1$ & $59 \pm 3$ & $73 \pm 2$ & $74 \pm 2$ & $71 \pm 2$ \\
\hline 3C 93.1 & $0345+33$ & 0.7 & & & & & $2.4 \pm 0.4$ & & & & & $59 \pm 3$ \\
\hline 4C 76.03 & $0404+76$ & $0.6^{+}$ & & & & & & & & & & \\
\hline OF247 & $0428+20$ & 0.49 & & & & & & & & & & \\
\hline 3C 119 & $0429+41$ & 0.9 & & & & $5.9 \pm 0.6$ & $8.8 \pm 0.4$ & & & & $49 \pm 2$ & $179 \pm 2$ \\
\hline 3C 138 & $0518+16$ & 3.25 & $7.2 \pm 0.25$ & $10.3 \pm 0.7$ & $9.9 \pm 0.8$ & $11.7 \pm 0.7$ & $9.9 \pm 0.4$ & $-11.1 \pm 0.0$ & $-11 \pm 2$ & $-13 \pm 2$ & $-11 \pm 2$ & $-7 \pm 2$ \\
\hline $3 \mathrm{C} 147$ & $0538+49$ & 2.58 & & & & & $1.2 \pm 0.4$ & & & & & $189 \pm 2$ \\
\hline $3 \mathrm{C} 186$ & $0740+38$ & 5.14 & & & & & & & & & & \\
\hline 3C 190 & $0758+14$ & 11.19 & & & & & & & & & & \\
\hline $3 \mathrm{C} 216$ & $0906+43$ & 33.44 & & & & & & & & & & \\
\hline $3 \mathrm{C} 237$ & $1005+07$ & 7.11 & & & & & $1.2 \pm 0.4$ & & & & & $18 \pm 4$ \\
\hline $3 \mathrm{C} 241$ & $1019+22$ & 3.82 & & & & & & & & & & \\
\hline 4C 31.38 & $1153+31$ & 3.96 & & & & & $1.9 \pm 0.4$ & & & & & $16 \pm 4$ \\
\hline $3 C 268.3$ & $1203+64$ & 5.28 & & & & $2.8 \pm 0.6$ & $3.0 \pm 0.4$ & & & & $-97 \pm 2$ & $-98 \pm 6$ \\
\hline ON343 & $1225+36$ & $0.23^{+}$ & & & & & & & & & & \\
\hline 3C 277.1 & $1250+56$ & 4.57 & & & & $3.0 \pm 0.6$ & $2.6 \pm 0.5$ & & & & $11 \pm 2$ & $7 \pm 6$ \\
\hline 4C 32.44 & $1323+32$ & $0.18^{+}$ & & & & & & & & & & \\
\hline $3 \mathrm{C} 286$ & $1328+30$ & 15.81 & $6.70 \pm 0.23$ & $10.9 \pm 0.7$ & $10.8 \pm 0.8$ & $11.7 \pm 0.7$ & $11.7 \pm 0.5$ & $35.0 \pm 0.0$ & $31 \pm 2$ & $33 \pm 2$ & $33 \pm 2$ & $33 \pm 2$ \\
\hline $3 \mathrm{C} 287$ & $1328+25$ & 0.43 & $0.65 \pm 0.12$ & $3.6 \pm 0.7$ & $3.7 \pm 0.8$ & $3.2 \pm 0.6$ & $3.3 \pm 0.4$ & $29.9 \pm 0.2$ & $105 \pm 2$ & $-32 \pm 2$ & $-15 \pm 2$ & $-6 \pm 2$ \\
\hline \multirow[t]{2}{*}{$4 \mathrm{C} 62.22$} & $1358+62$ & $0.17^{+}$ & & & & & & & & & & \\
\hline & $1413+34$ & 0.26 & & $3.1 \pm 0.7$ & & & & & $199 \pm 3$ & & & \\
\hline 3C 298 & $1416+06$ & 9.1 & & & & $1.8 \pm 0.6$ & $2.1 \pm 0.4$ & & & & $-42 \pm 3$ & $-30 \pm 4$ \\
\hline 3C 299 & $1419+41$ & 36.31 & & & & & & & & & & \\
\hline OQ172 & $1442+10$ & $0.1^{+}$ & $1.30 \pm 0.13$ & $4.5 \pm 0.9$ & $2.6 \pm 0.8$ & $2.2 \pm 0.6$ & & $-86.2 \pm 0.3$ & $212 \pm 4$ & $69 \pm 2$ & $76 \pm 3$ & \\
\hline 3C 303.1 & $1443+77$ & 1.28 & & & & & & & & & & \\
\hline 3C 305.1 & $1447+77$ & 7.86 & & & & & & & & & & \\
\hline 3C 309.1 & $1458+71$ & 9.25 & $1.20 \pm 0.13$ & $3.7 \pm 0.7$ & $2.4 \pm 0.7$ & $2.7 \pm 0.6$ & $2.5 \pm 0.4$ & $39.9 \pm 0.1$ & $104 \pm 2$ & $252 \pm 2$ & $247 \pm 2$ & $243 \pm 2$ \\
\hline \multirow[t]{2}{*}{ 3C 318} & $1517+20$ & 3.25 & & & $3.4 \pm 0.8$ & $6.6 \pm 0.6$ & $9.2 \pm 0.6$ & & & $60 \pm 2$ & $-14 \pm 2$ & $-24 \pm 2$ \\
\hline & $1600+33$ & $<0.4$ & & & & & & & & & & \\
\hline OS111 & $1607+26$ & $0.19^{+}$ & & & & & & & & & & \\
\hline 3C 343 & $1634+62$ & 11.48 & & & & & $1.4 \pm 0.4$ & & & & & $-104 \pm 3$ \\
\hline 3C 343.1 & $1637+62$ & 1.54 & & & & & & & & & & \\
\hline $3 \mathrm{C} 346$ & $1641+17$ & 27.01 & $2.20 \pm 0.14$ & & & & $1.8 \pm 0.4$ & $-82.7 \pm 0.1$ & & & & $-4 \pm 3$ \\
\hline 4C 39.56 & $1819+39$ & 3.20 & & & & & & & & & & \\
\hline $3 C 380$ & $1828+48$ & 55.62 & $0.45 \pm 0.12$ & & & & & $44.2 \pm 0.1$ & & & & \\
\hline 4C 29.56 & $1829+29$ & 12.40 & & & & & & & & & & \\
\hline 4C 11.69 & $2230+11$ & 9.7 & $1.6 \pm 0.13$ & $4.0 \pm 0.7$ & $3.6 \pm 0.8$ & $3.7 \pm 0.6$ & $3.6 \pm 0.4$ & $-61.1 \pm 0.1$ & $7 \pm 2$ & $48 \pm 2$ & $58 \pm 2$ & $60 \pm 2$ \\
\hline $3 C 454$ & $2249+18$ & 8.39 & $3.6 \pm 0.16$ & $5.8 \pm 0.7$ & $9.3 \pm 0.8$ & $11.2 \pm 0.7$ & $11.2 \pm 0.4$ & $53.4 \pm 0.1$ & $41 \pm 2$ & $87 \pm 2$ & $98 \pm 2$ & $101 \pm 2$ \\
\hline 3C 454.1 & $2248+71$ & 5.5 & & & & & & & & & & \\
\hline \multirow[t]{2}{*}{$3 \mathrm{C} 455$} & $2252+12$ & 11.75 & $2.10 \pm 0.14$ & $3.4 \pm 0.7$ & & & $1.5 \pm 0.5$ & $-7.5 \pm 0.2$ & $149 \pm 3$ & & & $151 \pm 8$ \\
\hline & $2342+82$ & $0.72^{+}$ & & & & & & & & & & \\
\hline
\end{tabular}

Table 3 is organized as follows: Col. 1, source name; Col. 2, other name; Col. 3, source linear size. Asterisks mark sources for which new redshifts have been obtained and new linear sizes were calculated; ${ }^{+}$signs mark sources with improved measurements of their angular sizes (Rossetti et al. 2005; 0319+12 Mantovani in prep.; 1442+10 Udomprasert et al. 1997); Cols. 4 to 8, percentage of polarised emission $m$; Cols. 9 to 13, position angle of the electric vector $\chi$. 
F. Mantovani et al.: Effelsberg polarimetry of CSS sources, Online Material $p 3$

Table 4. Depolarisation indices and RMs.

\begin{tabular}{|c|c|c|c|c|c|c|c|c|c|c|c|c|}
\hline Name & & $\bar{z}$ & 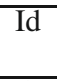 & $\begin{array}{r}\text { DP } \\
8.35 / 10.45 \\
\end{array}$ & $\begin{array}{r}\text { DP } \\
4.85 / 8.35 \\
\end{array}$ & $\begin{array}{r}\text { DP } \\
2.64 / 4.85 \\
\end{array}$ & $\begin{array}{r}\text { DP } \\
1.4 / 2.64 \\
\end{array}$ & $\begin{array}{r}\mathrm{RM}_{\text {obs }} \\
{\left[\mathrm{rad} \mathrm{m}^{-2}\right]}\end{array}$ & lm-1 & $\begin{array}{r}\mathrm{RM}_{\mathrm{obs}+\mathrm{TI}} \\
{\left[\mathrm{rad} \mathrm{m}^{-2}\right]} \\
\end{array}$ & $1 \mathrm{~m}-2$ & 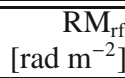 \\
\hline $3 \mathrm{C} 43$ & $0127+23$ & 1.46 & $\mathrm{Q}$ & 1.00 & & & & & & -69 & 0.9965 & -419 \\
\hline $3 \mathrm{C} 48$ & $0134+32$ & 0.37 & $\mathrm{Q}$ & 0.87 & 0.69 & & & -79 & 0.9994 & -64 & 0.9900 & -120 \\
\hline $3 \mathrm{C} 49$ & $0138+13$ & 0.62 & G & & & & & & & & & \\
\hline $3 \mathrm{C} 67$ & $0221+27$ & 0.31 & $\mathrm{G}$ & 1.05 & & & & -1 & 0.9999 & -67 & 0.9945 & -115 \\
\hline 4C 34.07 & $0223+34$ & $2.91 *$ & $\mathrm{Q}$ & & & & & & & & & \\
\hline \multirow[t]{2}{*}{ 4C 16.09} & $0316+16$ & $0.97 *$ & $\mathrm{Q}$ & & & & & & & & & -249 \\
\hline & $0319+12$ & 2.67 & Q & 1.16 & 1.09 & 0.89 & 0.07 & -12 & 0.9521 & -19 & 0.7698 & -164 \\
\hline 3C 93.1 & $0345+33$ & 0.24 & $\mathrm{G}$ & & & & & & & & & \\
\hline 4C 76.03 & $0404+76$ & 0.6 & $\mathrm{G}$ & & & & & & & & & \\
\hline OF247 & $0428+20$ & 0.22 & $\mathrm{G}$ & & & & & & & & & \\
\hline 3C 119 & $0429+41$ & 1.023 & Q & 0.66 & & & & & & 1928 & 0.9663 & 3900 \\
\hline 3C 138 & $0518+165$ & 0.76 & $\mathrm{Q}$ & 1.18 & 0.85 & 1.04 & 0.70 & -1 & 0.04 & -6 & 0.8786 & -19 \\
\hline 3C 147 & $0538+49$ & 0.55 & $\mathrm{Q}$ & & & & & & & & & \\
\hline $3 \mathrm{C} 186$ & $0740+38$ & 1.06 & Q & & & & & & & & & \\
\hline 3C 190 & $0758+14$ & 1.2 & Q & & & & & & & & & \\
\hline $3 \mathrm{C} 216$ & $0906+43$ & 0.67 & $\mathrm{Q}$ & & & & & & & & & \\
\hline 3C 237 & $1005+07$ & 0.88 & $\mathrm{G}$ & & & & & & & & & \\
\hline $3 \mathrm{C} 241$ & $1019+22$ & 1.62 & $\mathrm{G}$ & & & & & & & & & \\
\hline 4C 31.38 & $1153+31$ & 0.42 & $\mathrm{Q}$ & & & & & & & & & \\
\hline 3C 268.3 & $1203+64$ & 0.37 & G & 0.93 & & & & & & 114 & 0.9853 & 213 \\
\hline ON343 & $1225+36$ & $1.973 *$ & Q & & & & & & & & & \\
\hline 3C 277.1 & $1250+56$ & 0.32 & Q & 1.15 & & & & & & -59 & 0.9961 & -102 \\
\hline 4C 32.44 & $1323+32$ & 0.37 & $\mathrm{G}$ & & & & & & & & & \\
\hline $3 C 286$ & $1328+30$ & 0.85 & $\mathrm{Q}$ & 0.99 & 0.91 & 1.01 & 0.61 & & & & & \\
\hline $3 \mathrm{C} 287$ & $1328+25$ & 1.06 & $\mathrm{Q}$ & 0.97 & 1.16 & 0.97 & 0.18 & -148 & 0.9957 & -146 & 0.9954 & -618 \\
\hline $4 C 62.22$ & $\begin{array}{l}1358+62 \\
1413+34\end{array}$ & 0.43 & $\mathrm{G}$ & & & & 0.02 & & & & & \\
\hline 3C 298 & $1416+06$ & 1.44 & $\mathrm{Q}$ & 0.86 & & & & & & 96 & 0.9911 & 573 \\
\hline 3C 299 & $1419+41$ & 0.37 & $\mathrm{G}$ & & & & & & & & & \\
\hline OQ172 & $1442+10$ & 3.52 & $\mathrm{Q}$ & & 1.18 & 1.73 & 0.29 & -66 & 0.9994 & -71 & 0.9376 & -1451 \\
\hline 3C 303.1 & $1443+77$ & 0.2 & $\mathrm{G}$ & & & & & & & & & \\
\hline $3 \mathrm{C} 305.1$ & $1447+77$ & 1.13 & $\mathrm{G}$ & & & & & & & & & \\
\hline 3C 309.1 & $1458+71$ & 0.91 & $\mathrm{Q}$ & 1.08 & 0.89 & 1.54 & 0.32 & 60 & 0.9996 & 46 & 0.9830 & 169 \\
\hline 3C 318 & $\begin{array}{l}1517+20 \\
1600+33\end{array}$ & $\begin{array}{l}1.57 \\
1.1 *\end{array}$ & $\mathrm{G}$ & 0.72 & 0.52 & & & 498 & 0.9987 & 342 & 0.9995 & 2260 \\
\hline OS111 & $1607+26$ & $0.47 *$ & $\mathrm{G}$ & & & & & & & & & \\
\hline 3C 343 & $1634+62$ & 0.99 & $\mathrm{Q}$ & & & & & & & & & \\
\hline 3 C 343.1 & $1637+62$ & 0.75 & $\mathrm{G}$ & & & & & & & & & \\
\hline $3 \mathrm{C} 346$ & $1641+17$ & 0.16 & $\mathrm{G}$ & & & & & & & & & \\
\hline 4C 39.56 & $1819+39$ & 0.80 & $\mathrm{G}$ & & & & & & & & & \\
\hline 3C 380 & $1828+48$ & 0.69 & $\mathrm{Q}$ & & & & & & & & & \\
\hline 4C 29.56 & $1829+29$ & 0.84 & $\mathrm{G}$ & & & & & & & & & \\
\hline 4C 11.69 & $2230+11$ & 1.04 & $\mathrm{Q}$ & 1.03 & 0.97 & 1.10 & 0.40 & -39 & 0.9694 & -38 & 0.9894 & -157 \\
\hline 3C 454 & $2249+18$ & 1.76 & Q & 1.02 & & 0.62 & 0.62 & -88 & 1.000 & -88 & 0.9997 & -669 \\
\hline $3 \mathrm{C} 454.1$ & $2248+71$ & 1.84 & $\mathrm{G}$ & & & & & & & & & \\
\hline \multirow[t]{2}{*}{$3 \mathrm{C} 455$} & $2252+12$ & 0.54 & $\mathrm{G}$ & & & & 0.62 & -68 & 0.9402 & 82 & 0.9923 & 194 \\
\hline & $2342+82$ & 0.74 & $\mathrm{Q}$ & & & & & & & & & \\
\hline
\end{tabular}

Table 4 is organized as follows: Col. 1, source name; Col. 2, other name; Col. 3, redshift; redshifts marked with "** are taken from the following pubblications: 0223+34 Willott et al. (1998); 0316+16 Labiano et al. (2007); 1225+36 Lawrence et al. (1994); 1600+33 photometric redshift from Stanghellini et al. (2005); 1607+26 Stanghellini et al. (1993); Col. 4, optical identification. The source 3C 455 has been re-classified as galaxy by Hes et al. (1966); Cols. 5 to 8, depolarisation indices DP; Col. 9, rotation measure RM derived from our new Effelsberg observations; Col. 10, ls-1: the least square fit of the linear regression of the values in Col. 9; Col. 11, rotation measure RM derived from our new Effelsberg observations, complemented with data from Tabara \& Inoue (1980); Col. 12, 1s-2: the least square fit of the linear regression of the RM values in Col. 11; Col. 13, rest frame $\mathrm{RM}$ calculated using the $\mathrm{RM}_{\mathrm{obs}+\mathrm{TI}}$ values. 
F. Mantovani et al.: Effelsberg polarimetry of CSS sources, Online Material p 4

\section{Appendix 1}

In this Appendix, we present plots of the fractional polarisation $m$ derived form our Effelsberg observations complemented with those extracted from the NVSS at $1.4 \mathrm{GHz}$ and those taken from Tabara \& Inoue (1980). The values of $m$ predicted by the models of Burn and Tribble are shown for comparison. Also plotted are polarisation angles versus $\lambda^{2}$ with their corresponding linear best fit. 
F. Mantovani et al.: Effelsberg polarimetry of CSS sources, Online Material p 5
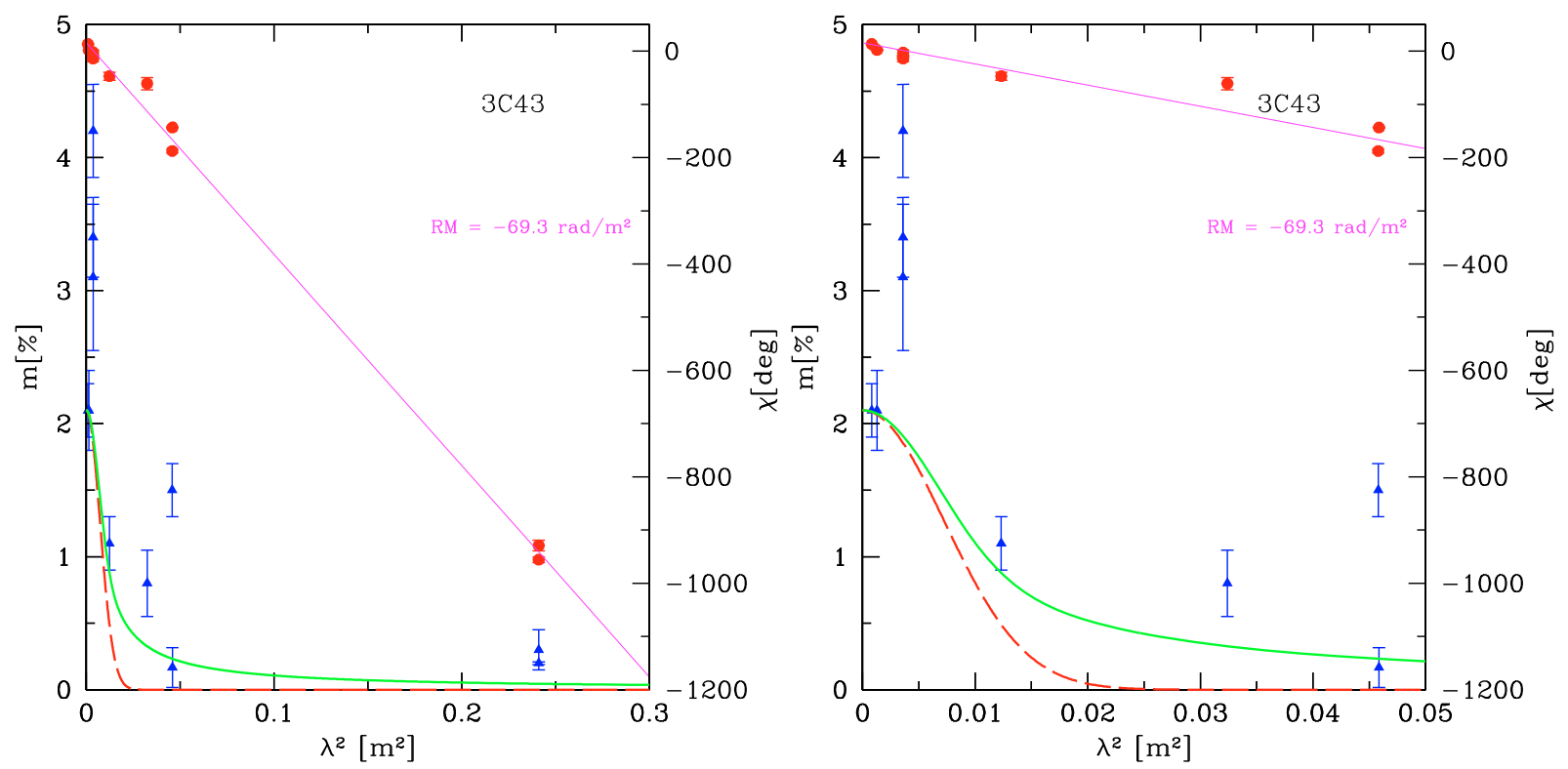

Fig. A.1. Position angles of the electric field vector $\chi$ in deg (dots) and fractional polarisation $m$ in $\%$ (triangles) versus $\lambda^{2}$ in $\mathrm{m}^{2}$ for the source $3 \mathrm{C} 43$, for the full range (left), and for a narrow range (right) of wavelengths. The solid line represents the Tribble model, the dashed line the Burn model.
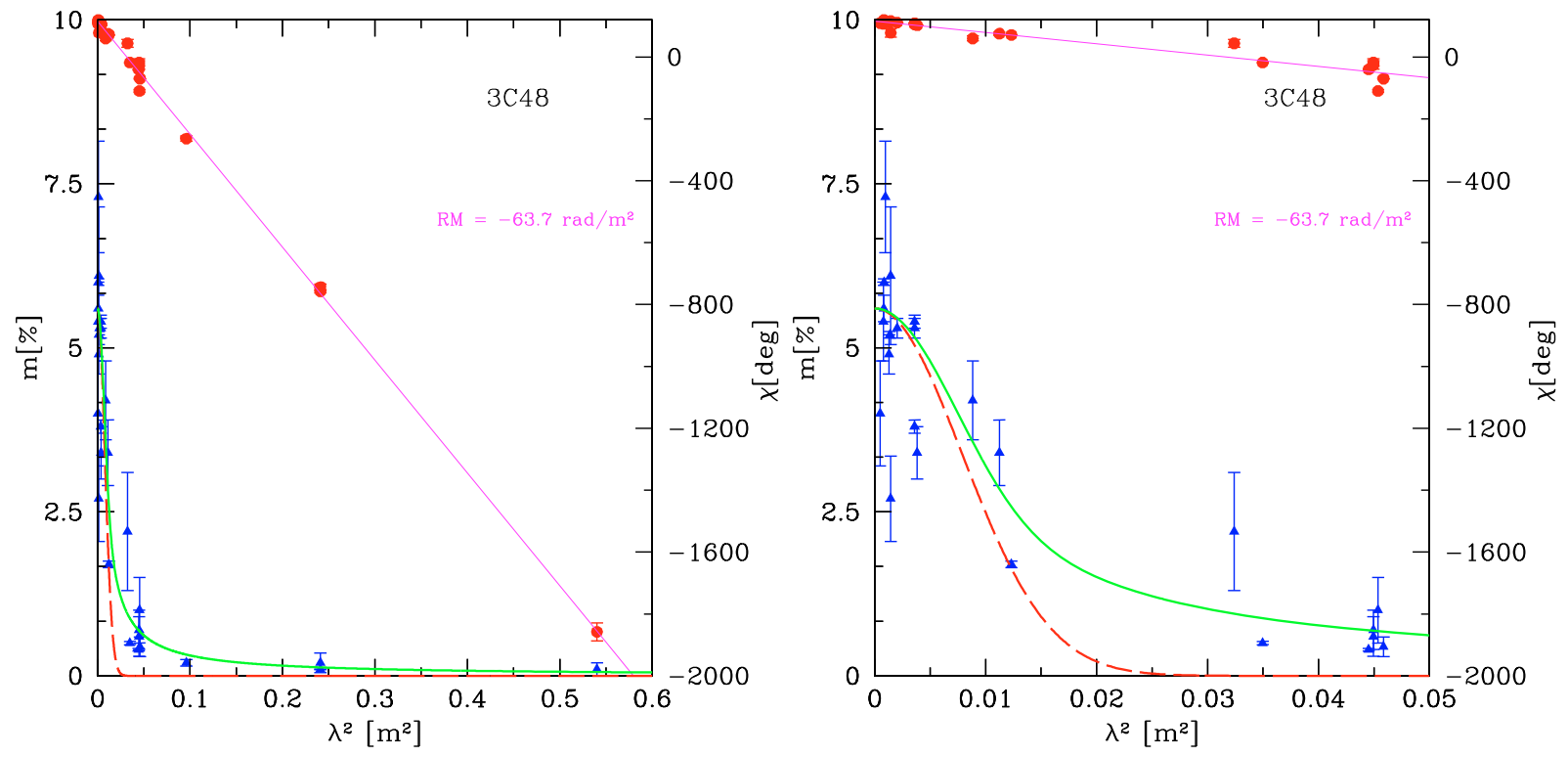

Fig. A.2. Position angles $\chi$ and fractional polarisation $m$ for the source $3 C$ 48. Layout as in Fig. A.1. 
F. Mantovani et al.: Effelsberg polarimetry of CSS sources, Online Material p 6

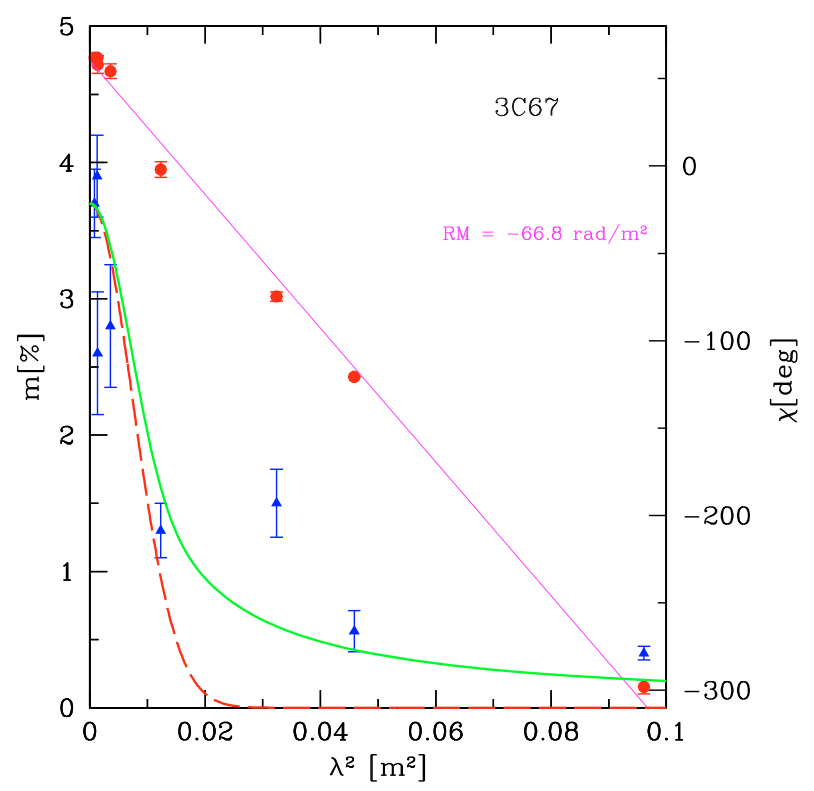

Fig. A.3. Position angles $\chi$ and fractional polarisation $m$ for the source 3C 67. Layout as in Fig. A.1.

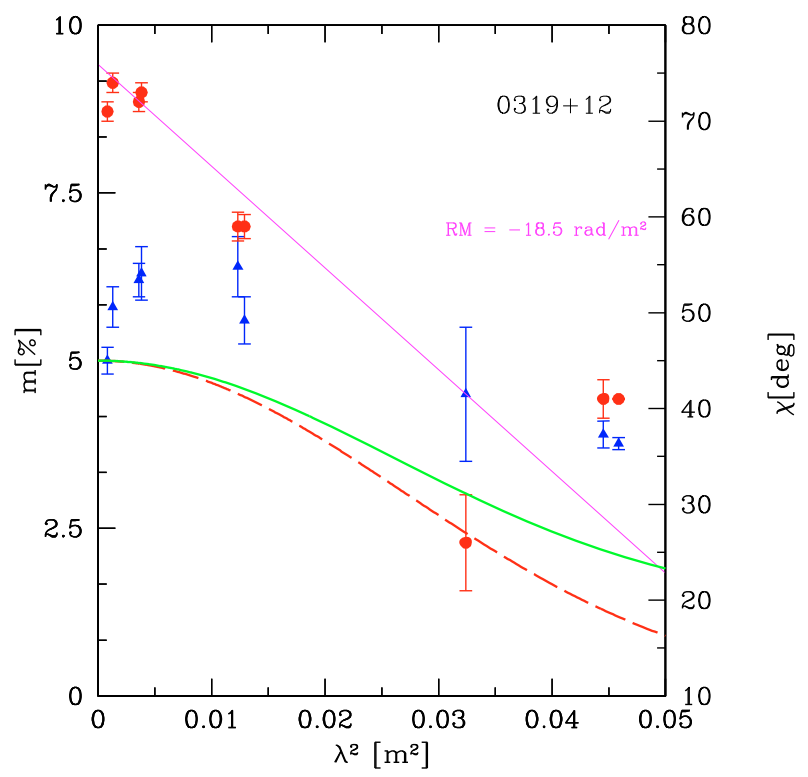

Fig. A.4. Position angles $\chi$ and fractional polarisation $m$ for the source 0319+12. Layout as in Fig. A.1. 
F. Mantovani et al.: Effelsberg polarimetry of CSS sources, Online Material $p 7$
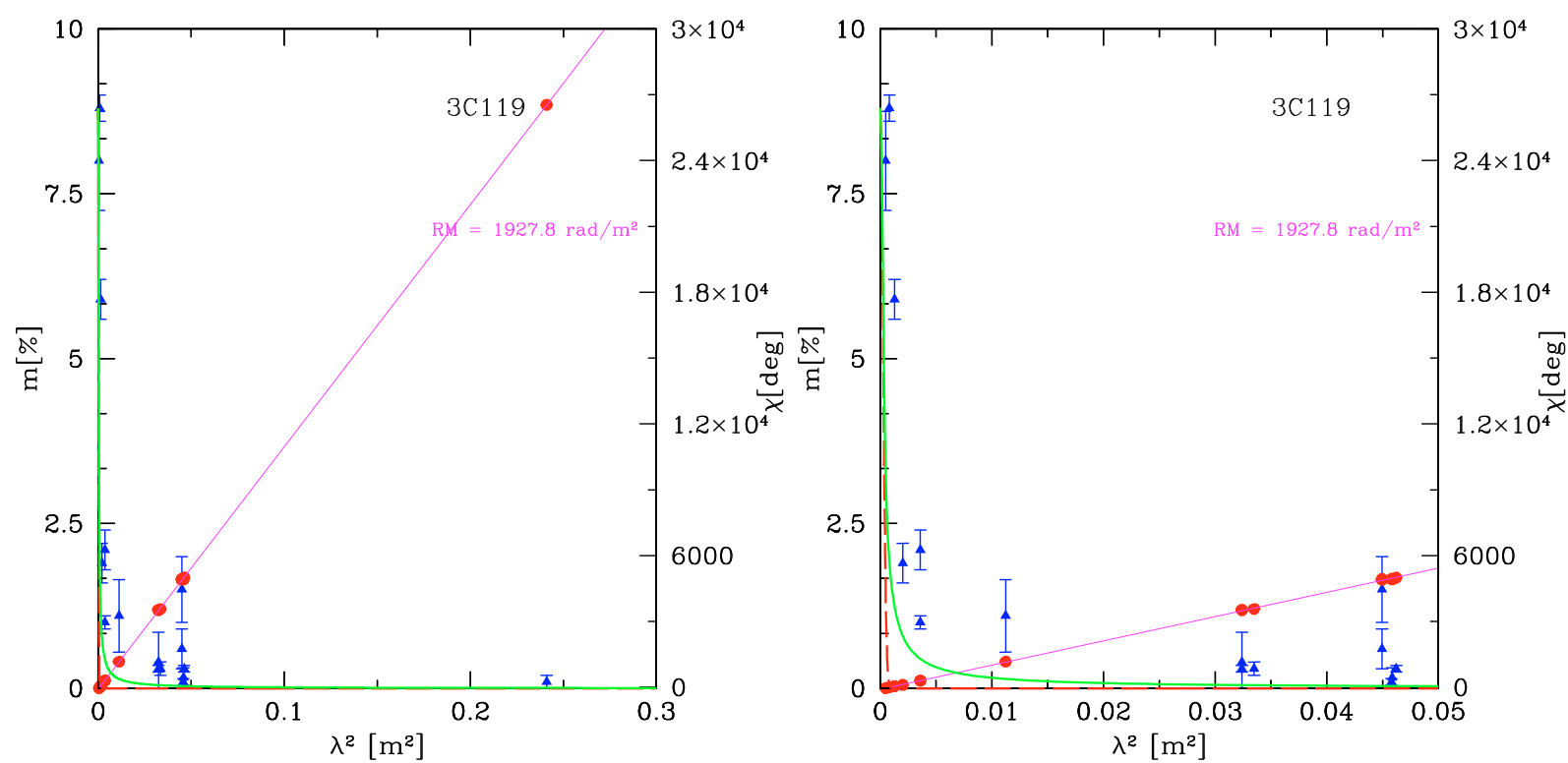

Fig. A.5. Position angles $\chi$ and fractional polarisation $m$ for the source $3 C$ 119. Layout as in Fig. A.1.
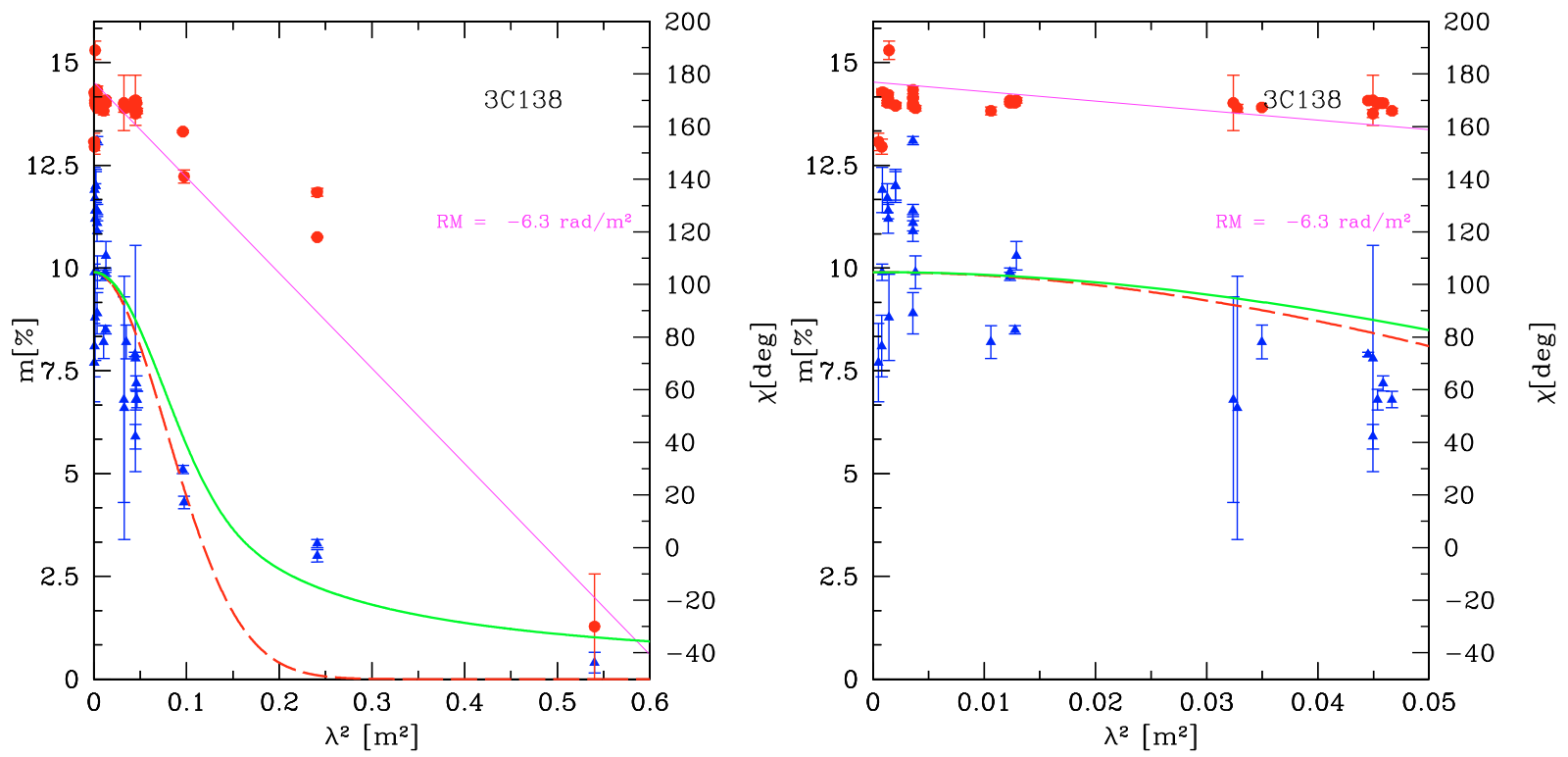

Fig. A.6. Position angles $\chi$ and fractional polarisation $m$ for the source 3C 138. Layout as in Fig. A.1. 
F. Mantovani et al.: Effelsberg polarimetry of CSS sources, Online Material $p 8$

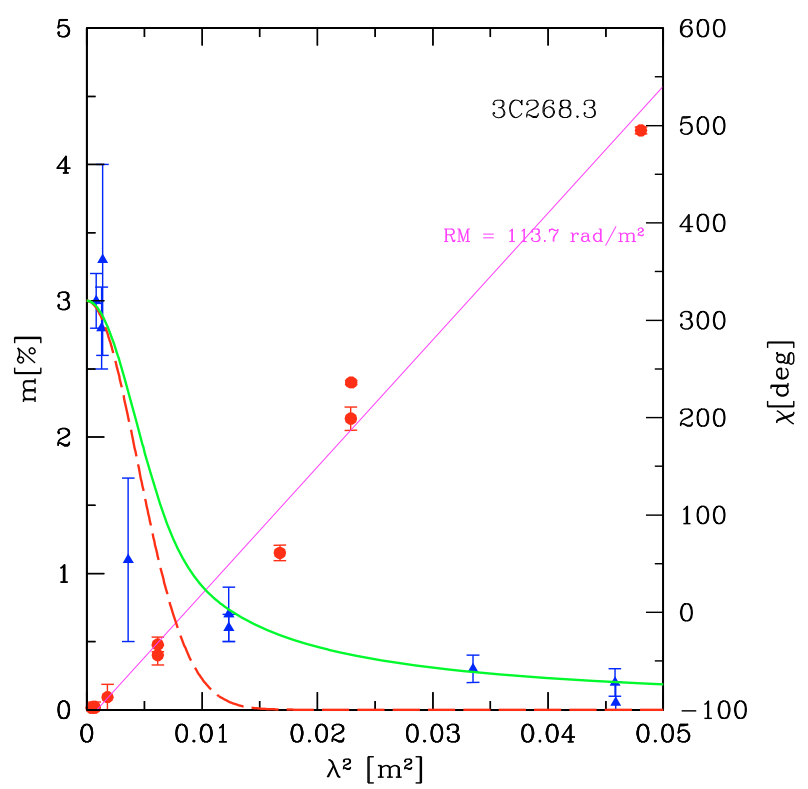

Fig. A.7. Position angles $\chi$ and fractional polarisation $m$ for the source 3C 268.3. Layout as in Fig. A.1. 
F. Mantovani et al.: Effelsberg polarimetry of CSS sources, Online Material $p 9$
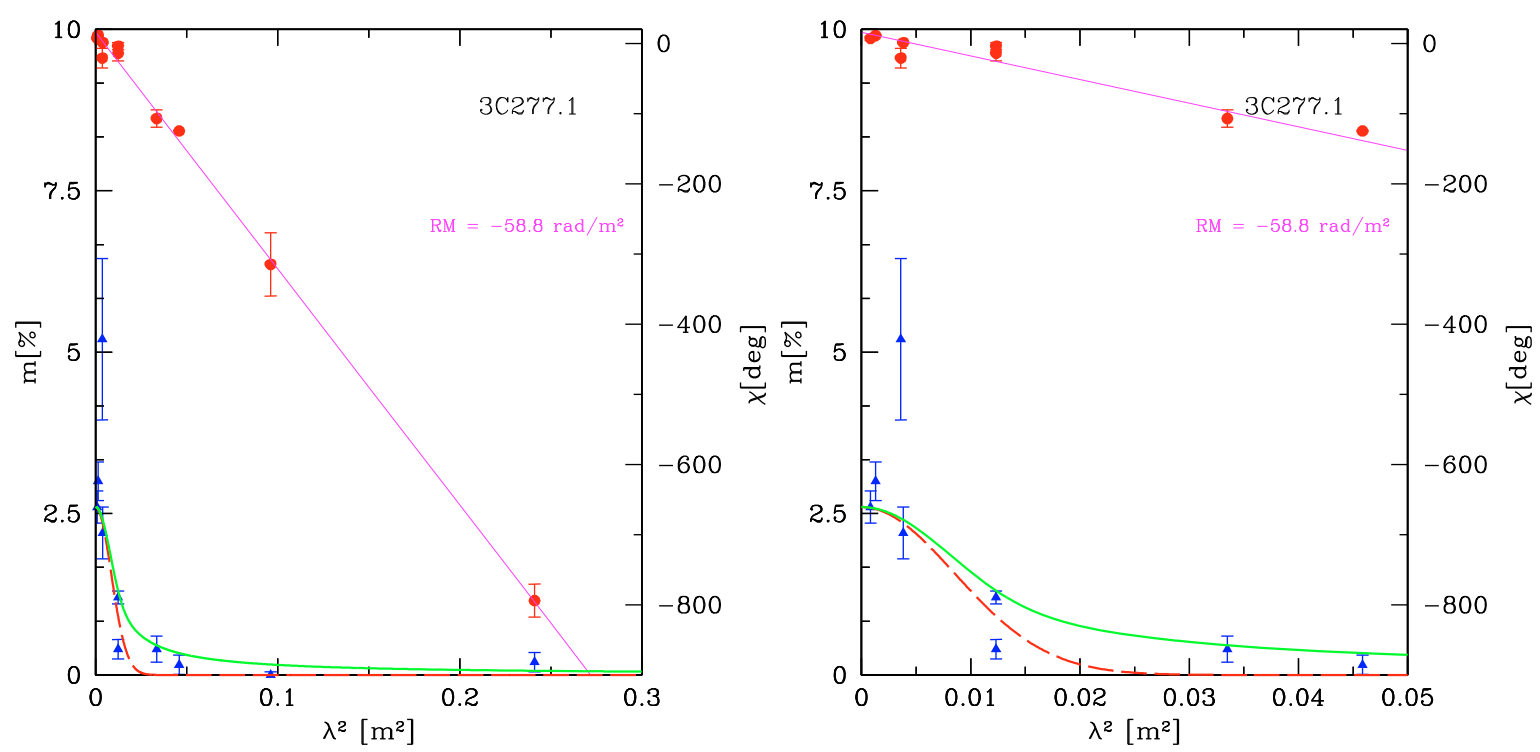

Fig. A.8. Position angles $\chi$ and fractional polarisation $m$ for the source 3C 277.1. Layout as in Fig. A.1.
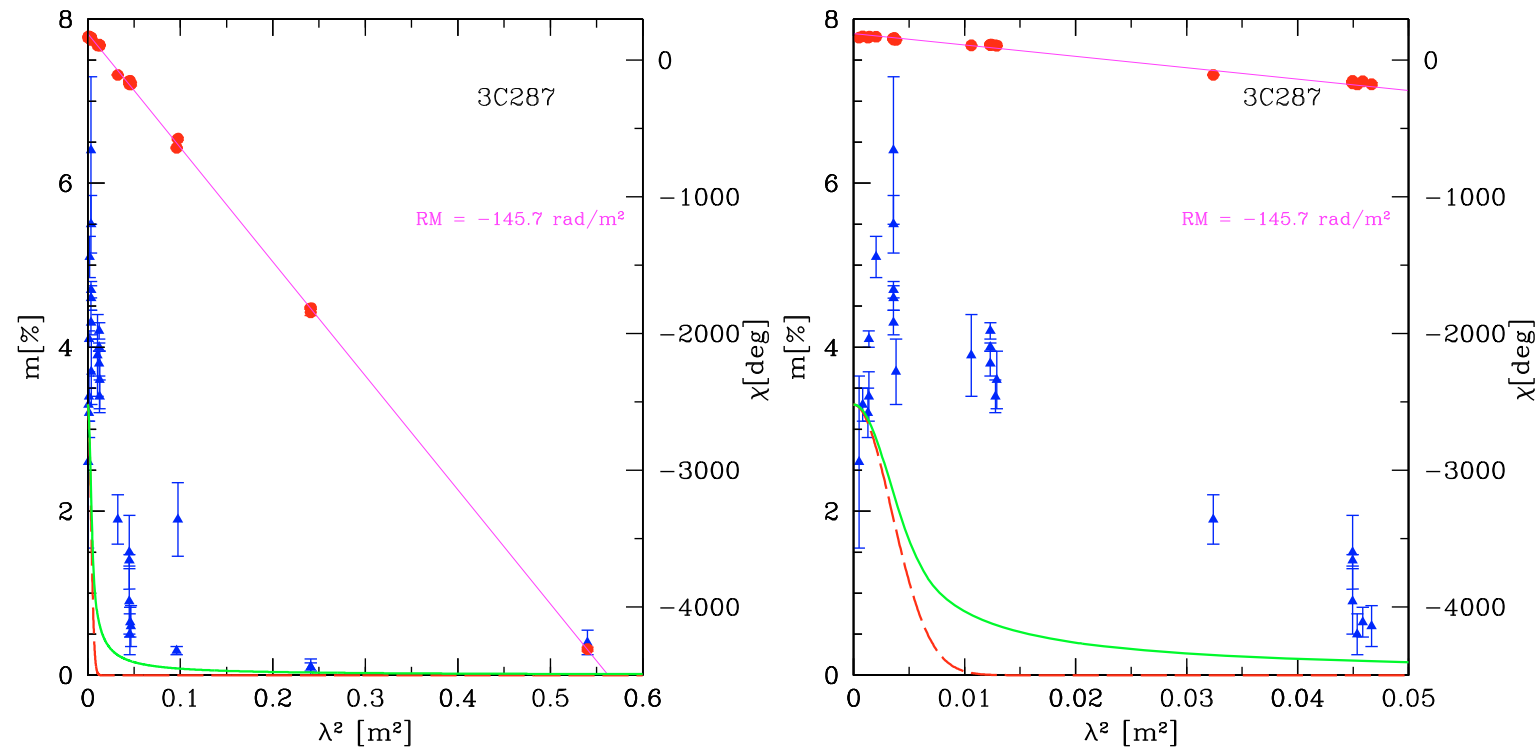

Fig. A.9. Position angles $\chi$ and fractional polarisation $m$ for the source 3 C 287. Layout as in Fig. A.1.
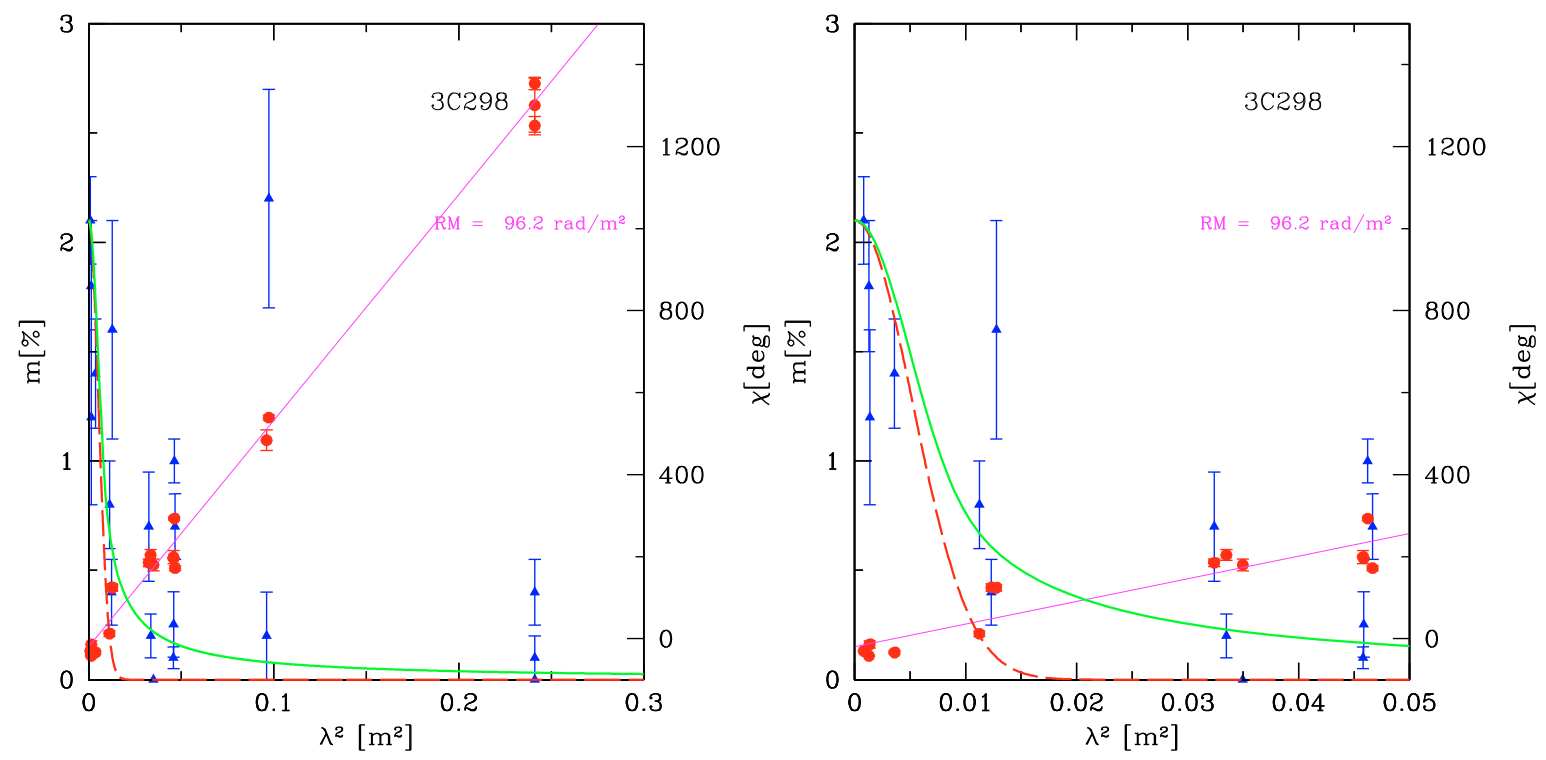

Fig. A.10. Position angles $\chi$ and fractional polarisation $m$ for the source 3C 298. Layout as in Fig. A.1. 
F. Mantovani et al.: Effelsberg polarimetry of CSS sources, Online Material p 10

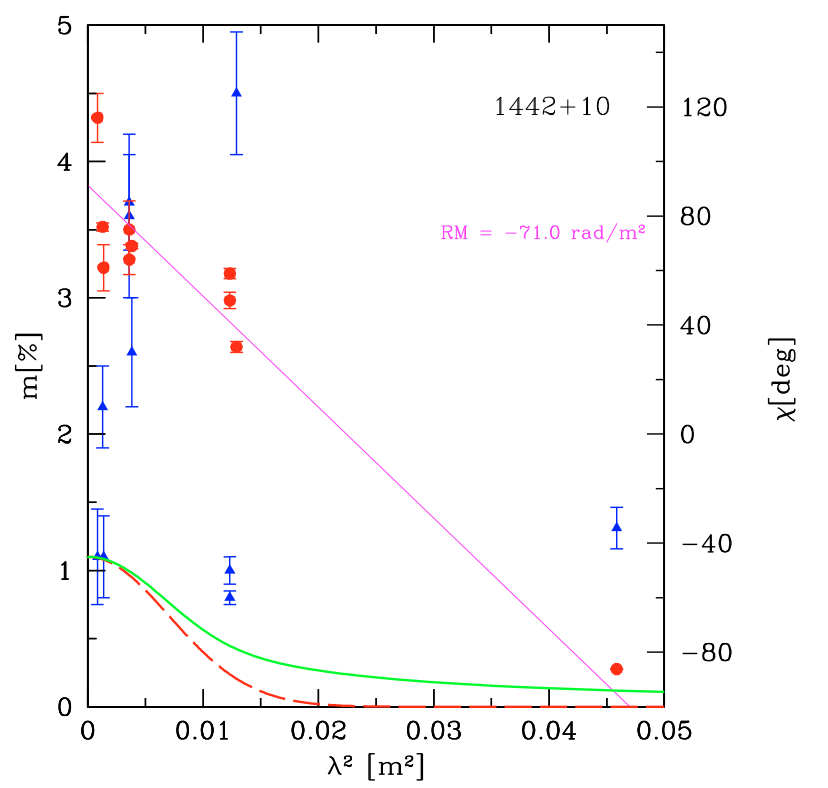

Fig. A.11. Position angles $\chi$ and fractional polarisation $m$ for the source 1442+10. Layout as in Fig. A.1. 
F. Mantovani et al.: Effelsberg polarimetry of CSS sources, Online Material p 11
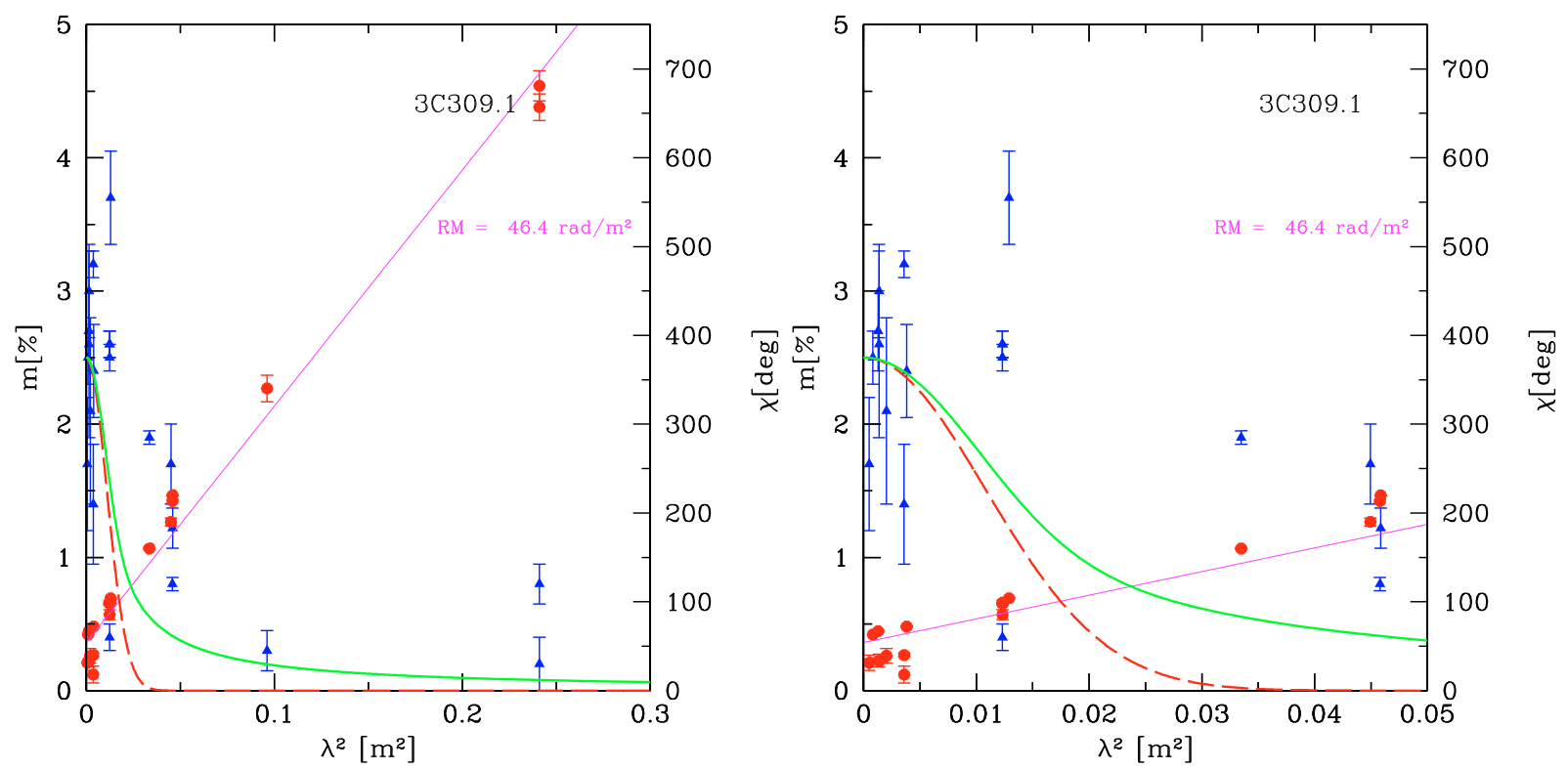

Fig. A.12. Position angles $\chi$ and fractional polarisation $m$ for the source 3C 309.1. Layout as in Fig. A.1.
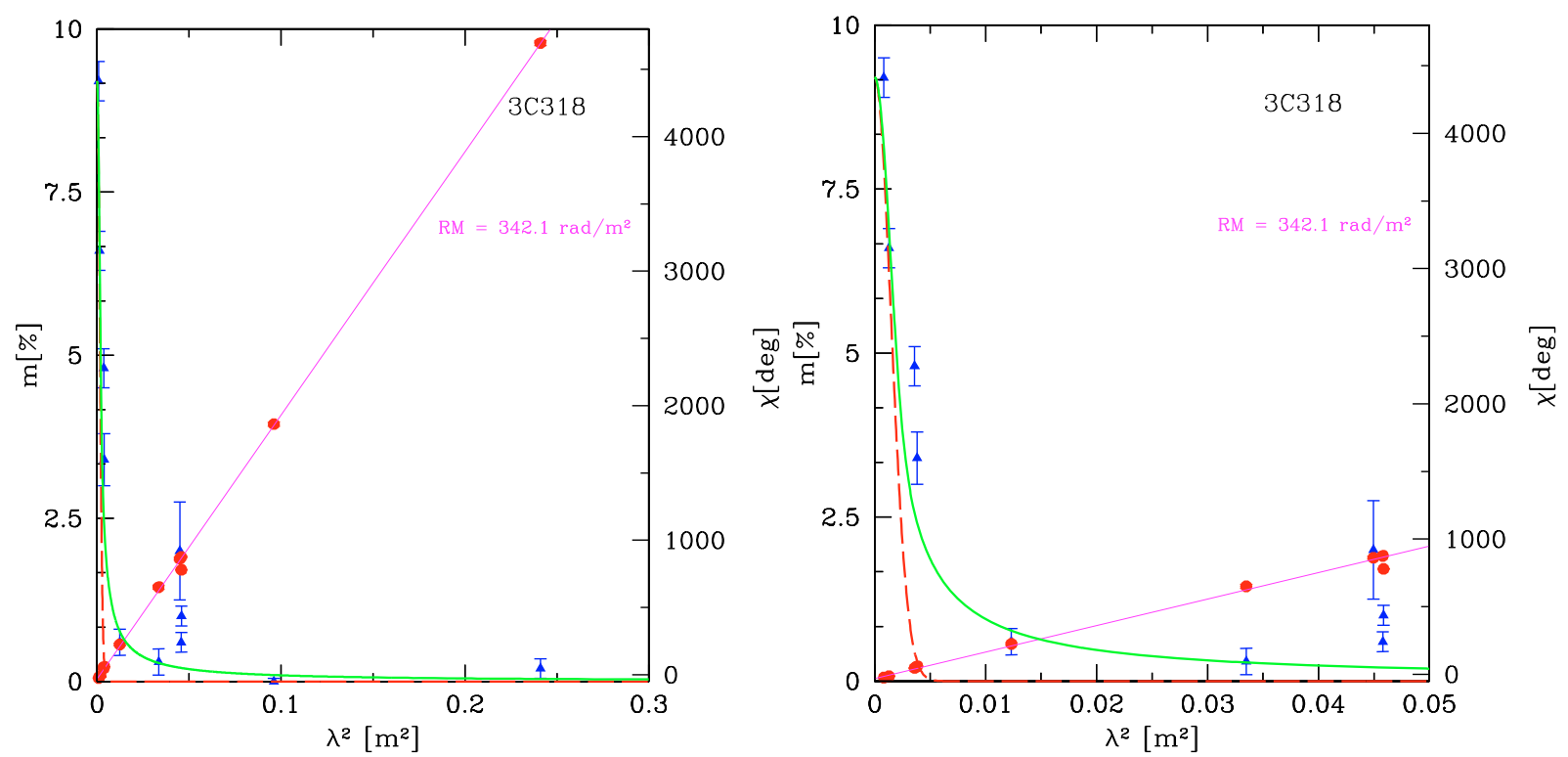

Fig. A.13. Position angles $\chi$ and fractional polarisation $m$ for the source 3C 318. Layout as in Fig. A.1. 
F. Mantovani et al.: Effelsberg polarimetry of CSS sources, Online Material p 12
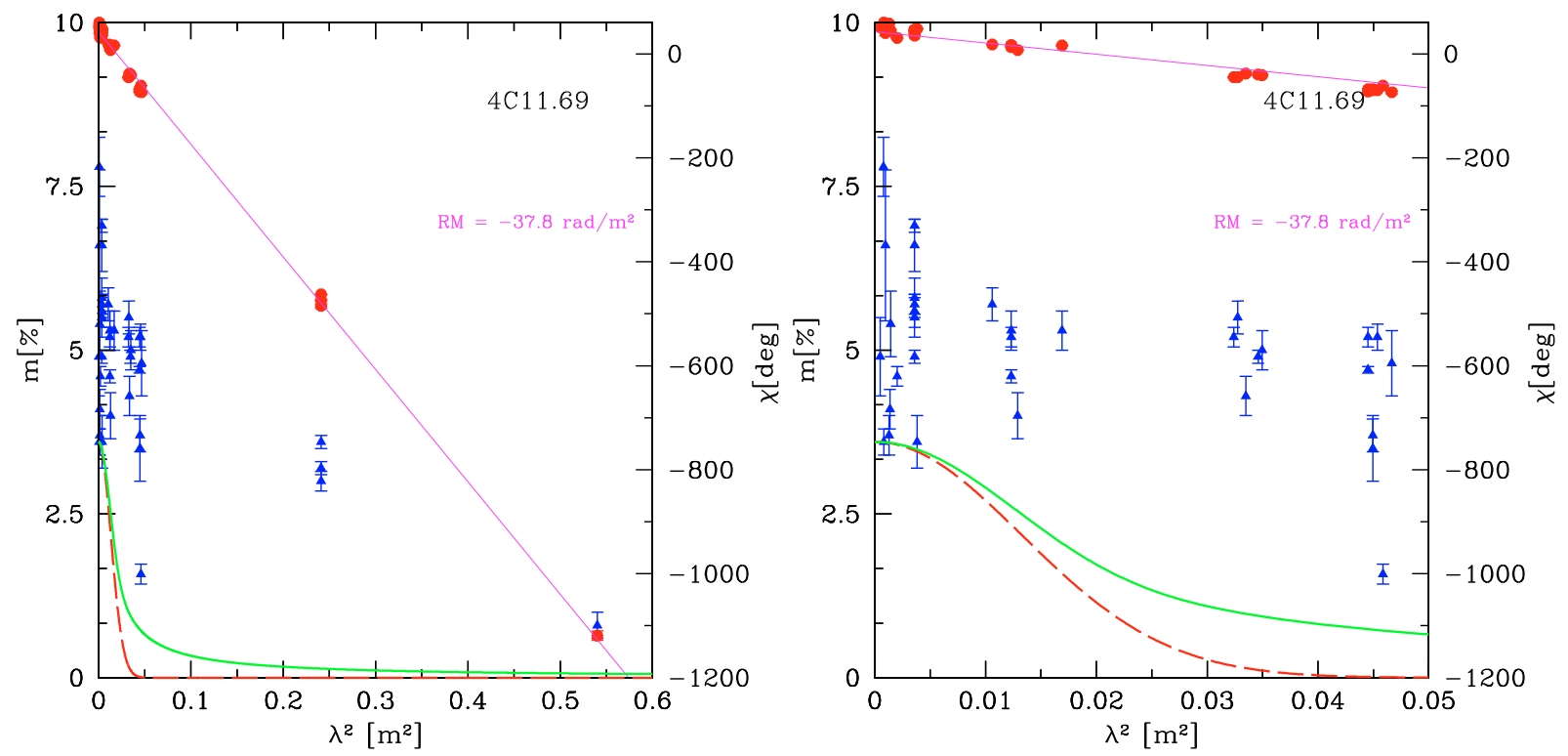

Fig. A.14. Position angles $\chi$ and fractional polarisation $m$ for the source 4C 11.69. Layout as in Fig. A.1.
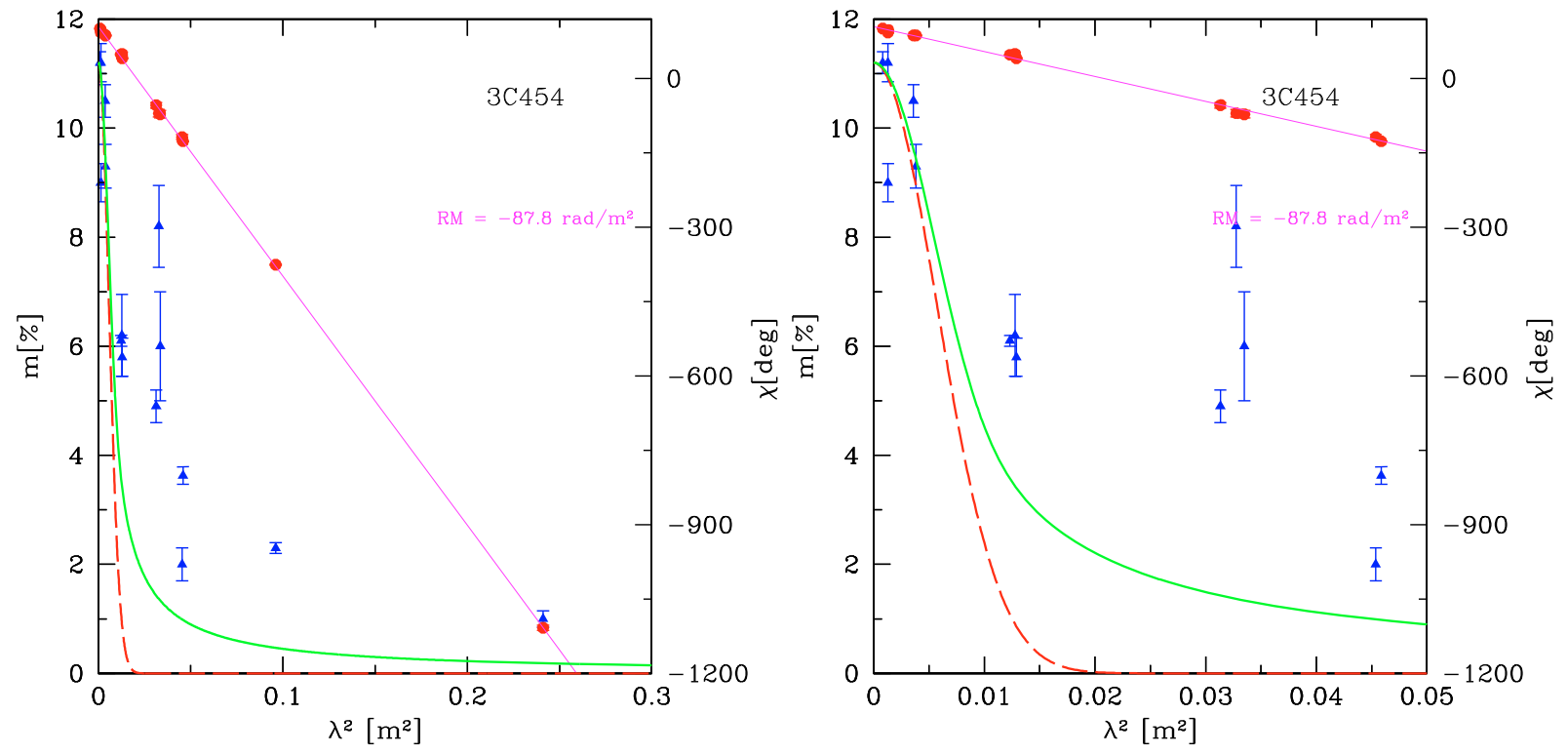

Fig. A.15. Position angles $\chi$ and fractional polarisation $m$ for the source $3 \mathrm{C} 454$. Layout as in Fig. A.1. 
F. Mantovani et al.: Effelsberg polarimetry of CSS sources, Online Material p 13
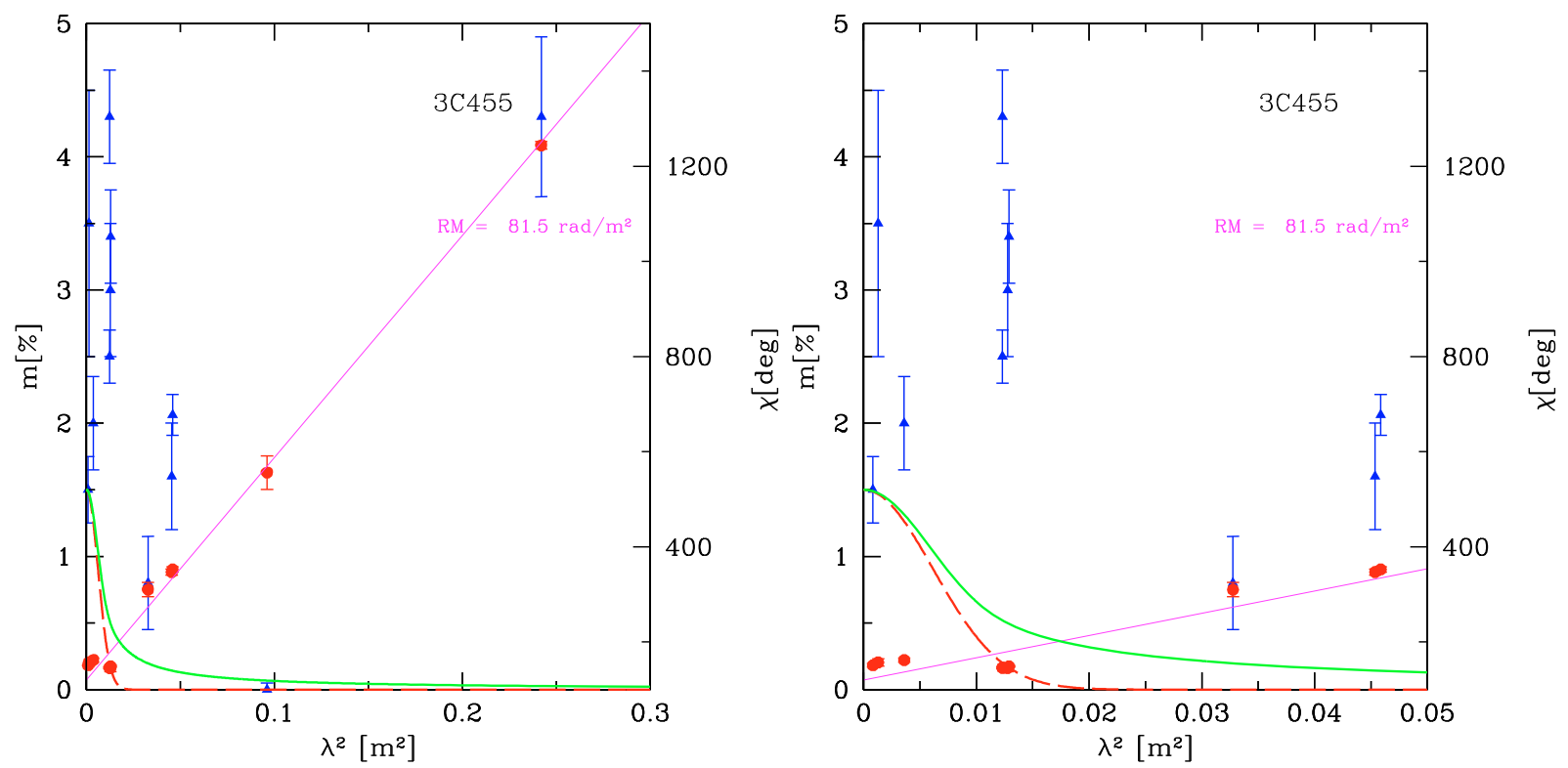

Fig. A.16. Position angles $\chi$ and fractional polarisation $m$ for the source $3 C$ 455. Layout as in Fig. A.1. 


\section{Appendix 2}

\subsection{Sources following the Tribble model}

The model proposed by Tribble reproduces the data of about one third of our sample, namely of 3C 48, 3C 67, 3C 119, 3C 138, 3C 268.3, 3C 277.1, and 3C 318. Sources such as 3C 48, 3C 67, 3C 138, and 3C 277.1 also show RM $<\lesssim$ few $10 \mathrm{rad} \mathrm{m}^{-2}$, which is an indication of an unresolved foreground screen, presumably the halo of our own Galaxy.

The source 3C 119, which has a very high RM and thus a fast decline in its fractional polarisation, might already be affected by significant depolarisation at wavelengths shorter than $2.8 \mathrm{~cm}$. A higher $m_{0}$ would lead to depolarisation according to the Tribble law.

\subsection{Sources with an indication of repolarisation}

Eight sources (3C 43, 0319+12, 3C 287, 1442+10, 3C 309.1, 4C $11.69,3 \mathrm{C} 454$, and 3C 455) show indications of repolarisation, i.e., an increase in fractional polarisation with decreasing frequency, generally at short wavelengths $\lesssim 10 \mathrm{~cm}$; this corresponds to a relatively strong increase in fractional polarisation followed by a decline that can still be described by the Tribble law. This effect is visible despite possible instrumental effects of different telescopes and also considering that observations were made at different epochs. In three of these sources (3C 309.1, 4C 11.69, 3C 454), the repolarisation effect is indeed confused with possible time variability. Focusing on our simultaneous measurements only, we still find constant or slightly increasing fractional polarisation with increasing wavelength, which is not predicted by any depolarisation model. In particular, the galaxy 3C 455 shows a measured repolarisation greater than the $3 \sigma$ level between $10.45 \mathrm{GHz}$ and $2.64 \mathrm{GHz}$ together with a small value of the
$\mathrm{RM}_{\mathrm{rf}}\left(194 \mathrm{rad} \mathrm{m}^{-2}\right)$, which is an indication of the influence of a foreground screen, possibly an extended cloud with [OII] emission detected by Hes et al. (1996). A plausible mechanism would be the effect of shear layers caused by the interaction between the surface of an expanding source and the surrounding medium (Burn 1966).

The source 3C 455 was observed with the VLA at $8.35 \mathrm{GHz}$ by Bogers et al. (1994). It shows a triple structure with the indication of a jet joining the core with the south western lobe. The three components are almost aligned along the source major axis extending up to about $4 "$. However, 3C 455 appears slightly resolved by the NVSS, which has a restoring beam of $45^{\prime \prime}$, suggesting that the three components imaged by Bogers et al. are actually embedded in a more extended region of low brightness emission. This can be seen in the image available in the VLA Low-Frequency Sky Survey (VLSS; Cohen et al. 2007) at $74 \mathrm{MHz}$, which shows an even more extended structure of about $2^{\prime}$ in size. The existence of this extended emission is supported by the source spectral index, which indicates an upturn towards higher flux density above $\sim 100 \mathrm{MHz}$. Therefore, a second possible interpretation is that by observing at $2.64 \mathrm{GHz}$ or lower frequencies, we have integrated the flux density and polarised flux density from that region. At $10.45 \mathrm{GHz}$, the steep spectrum extended structure is below the detection limit.

A similar case of repolarisation at a lower frequency was pointed out by Montenegro-Montes et al. (2008) for the source $1159+01$.

\subsection{Polarisation variability}

Finally, 3C 298 could exhibit time variability in its fractional polarisation. Unfortunately, the current data base does not provide a sufficient number of simultaneous measurements to prove this effect, which was reported for example by Aller et al. (2003) for the source 3C 147. 